\title{
Phase engineering of two-dimensional transition metal dichalcogenides
}

\author{
Yao $\mathrm{Xiao}^{1}$, Mengyue $\mathrm{Zhou}^{2}$, Jinglu $\mathrm{Liu}^{2}$, Jing $\mathrm{Xu}^{2}$ and $\mathrm{Lei} \mathrm{Fu}^{1,2^{*}}$
}

\begin{abstract}
Two-dimensional (2D) transition metal dichalcogenides (TMDs) have gained much attention in virtue of their various atomic configurations and band structures. Apart from those thermodynamically stable phases, plenty of metastable phases exhibit interesting properties. To obtain 2D TMDs with specific phases, it is important to develop phase engineering strategies including phase transition and phaseselective synthesis. Phase transition is a conventional method to transform one phase to another, while phase-selective synthesis means the direct fabrication of the target phases for 2D TMDs. In this review, we introduce the structures and stability of 2D TMDs with different phases. Then, we summarize the detailed processes and mechanism of the traditional phase transition strategies. Moreover, in view of the increasing demand of high-phase purity TMDs, we present the advanced phase-selective synthesis strategies. Finally, we underline the challenges and outlooks of phase engineering of $2 \mathrm{D}$ TMDs in two aspects-high phase purity and excellent controllability. This review may promote the development of controllable phase engineering for 2D TMDs and even other 2D materials toward both fundamental studies and practical applications.
\end{abstract}

Keywords: phase engineering, transition metal dichalcogenides, phase transition, phase-selective synthesis

\section{INTRODUCTION}

Beyond graphene, transition metal dichalcogenides (TMDs) have become a series of outstanding twodimensional (2D) materials for the variation of their structures and properties [1-10]. 2D TMDs with diverse band structures are essential to both fundamental research and applications. In detail, 2D TMDs with suitable bandgaps can be utilized for high-performance logic circuits and broadband photodetectors [1,11], and others exhibit novel physical properties including super- conductivity, ferroelectricity, ferromagnetism and quantum spin Hall (QSH) effect [12-20]. Owing to the different symmetry, TMDs with different phases vary in the electron density of state (DOS) and charge distribution, as well as phonon DOS (PDOS), which directly affect their electrical, optical, magnetic, thermal and even mechanical properties [21-25].

Theoretical studies demonstrate a number of TMD structures with remarkable properties, and their stability varies greatly [26-29]. Some metastable phases are hardly obtained in the ambient condition. On the basis of close connection among TMD structures, stability and properties, it is vital to exploit effective pathways toward the specific phases. Therefore, phase engineering for TMDs has gained wide attention, which can open up a new territory for modulating the structure and properties of TMDs [30,31].

Here, in view of recent progress, we reviewed phase engineering of 2D TMDs. Initially, we introduced several types of TMD structures, and summarized their stability and properties. Then, we expounded phase engineering strategies which were classified into two types-phase transition and phase-selective synthesis, in accordance with the different process (Fig. 1). Specifically, phase transition is the transformation from one phase to another, where a stable TMD generally turns into a metastable one. Phase-selective synthesis focuses on the direct fabrication of high-purity phase TMDs or heterostructures rather than the transformation process. We presented the promoting factors and the corresponding feature and mechanism for the phase transition, including intercalation, charge transfer, external irradiation, thermal treatment and stress induction. For phaseselective synthesis, we analyzed the influence of different reaction conditions such as the precursor, temperature, atmosphere and extra assisted medium on the as-

\footnotetext{
${ }^{1}$ The Institute for Advanced Studies (IAS), Wuhan University, Wuhan 430072, China

${ }^{2}$ College of Chemistry and Molecular Sciences, Wuhan University, Wuhan 430072, China

* Corresponding author (email: leifu@whu.edu.cn)
} 


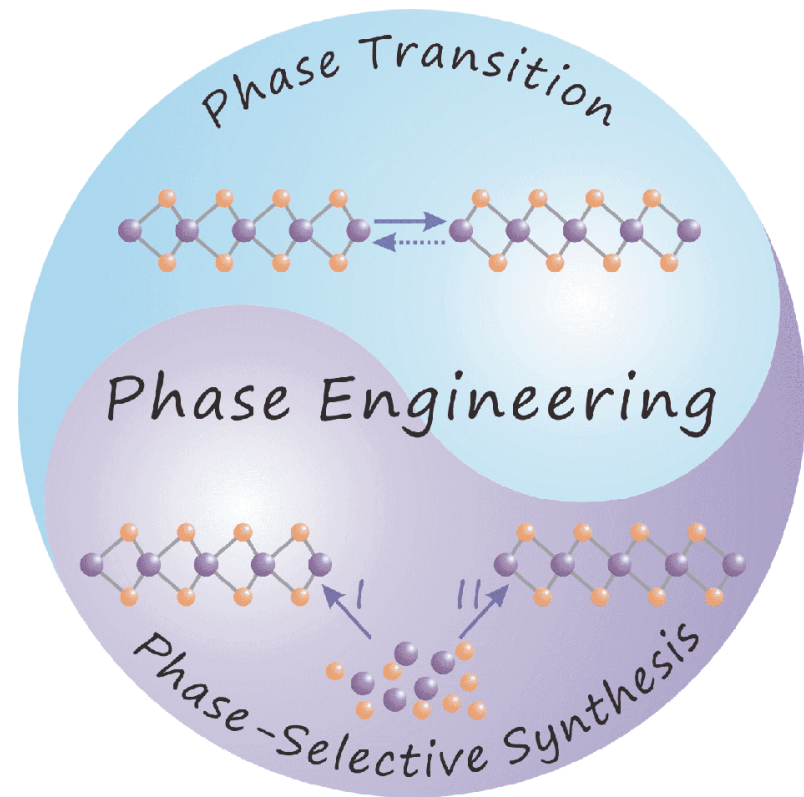

Figure 1 Schematic illustration of phase engineering of 2D TMDs.

synthesized 2D TMDs. Finally, we summarized the merits and demerits for both the phase transition and phaseselective synthesis strategies. We also pointed out the problems in phase engineering at present and the possible trend in the future. We believe that phase engineering of 2D TMDs is promising for the discovery of novel physical phenomena and high-performance devices.

\section{STRUCTURES AND STABILITIES OF TMDs}

With the variation of the oxidation states of transition metals, 2D TMDs exhibit diverse structures. According to the atomic configurations, monolayer 2D TMDs generally present two basic phases-the trigonal prismatic and the octahedral phases, referring to the $1 \mathrm{H}$ and $1 \mathrm{~T}$ phase with $D_{3 \mathrm{~h}}$ and $D_{3 \mathrm{~d}}$ symmetry, respectively (Fig. $2 \mathrm{a}$ and b) $[1,21]$. In addition, $1 \mathrm{~T}$ phase TMDs derive distorted structures (1 $\mathrm{T}^{\prime \prime}$ and $1 \mathrm{~T}^{\prime \prime}$ phases) (Fig. $2 \mathrm{c}$ and $\mathrm{d}$ ), according to the different distortions [32]. The different stacking arrangements of $1 \mathrm{H}$ phase layers result in $2 \mathrm{H}$ and $3 \mathrm{R}$ phases (Fig. 2e and f). Besides these polymorphs, the transformation from $1 \mathrm{~T}^{\prime}$ phase to $T_{d}$ phase also derives from the symmetry change of stacked $1 \mathrm{~T}^{\prime}$ layers [2]. Different atomic configurations of TMDs originate from the different situation of the filling of $\mathrm{d}$ orbitals of transition metals. In addition to the different groups of transition metals, the stability of $2 \mathrm{D}$ TMDs is ascribed to the filling of $\mathrm{d}$ orbitals [21]. Therefore, 2D TMDs with different phases show the variations of thermodynamic stability [27]. Fig. 2g lists the layered TMDs together with the corresponding structures and stability. In detail, group IVB $\left(\mathrm{d}^{0}\right)$ and group VIII $\left(\mathrm{d}^{6}\right)$ TMDs only have octahedral structures. Most of group VIB $\left(\mathrm{d}^{2}\right)$ TMDs present trigonal prismatic phases, and group VB $\left(\mathrm{d}^{1}\right)$ TMDs could exhibit both trigonal prismatic and octahedral structures, whereas group VIIB $\left(d^{3}\right)$ TMDs generally show distorted octahedral structures. Taking Mo- and W-based TMDs as

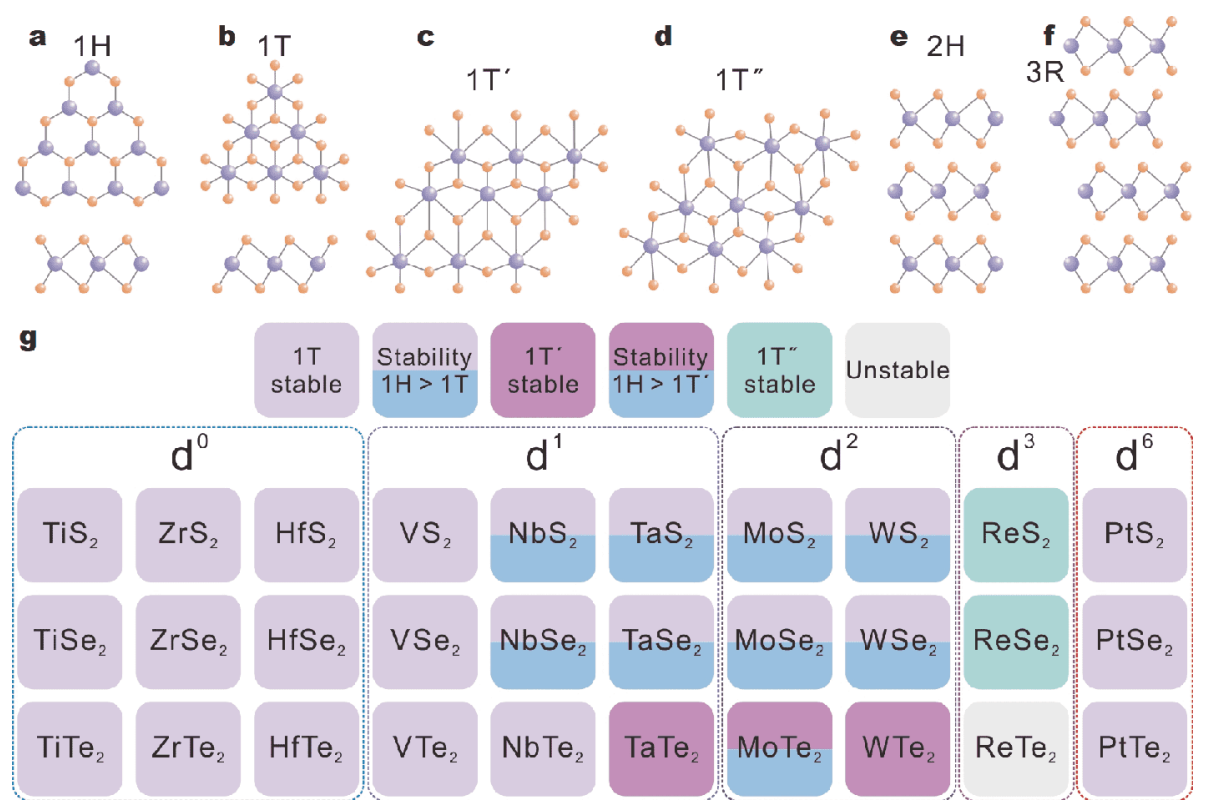

Figure 2 Structures and stability of 2D TMDs. (a-f) Different phases of 2D TMDs: (a) $1 \mathrm{H}$, (b) 1T, (c) 1T', (d) $1 \mathrm{~T}^{\prime \prime}$, (e) $2 \mathrm{H}$ and (f) $3 \mathrm{R}$ phases. (g) Summary of structures and stability of 2D TMDs including group IVB, VB, VIB, VIIB and VIII. 
examples, the trigonal prismatic phases of $(\mathrm{Mo}, \mathrm{W}) \mathrm{S}_{2}$, $(\mathrm{Mo}, \mathrm{W}) \mathrm{Se}_{2}$ and $\mathrm{MoTe}_{2}$ are much more stable than the octahedral ones of those, whereas the situation is completely opposite for $\mathrm{WTe}_{2}$, in accordance with the ground state energy differences [33]. It means that $\mathrm{WTe}_{2}$ tends to become a $1 \mathrm{~T}^{\prime}$ phase rather than a $1 \mathrm{H}$ phase at the ground state.

Variation of 2D TMD phases provides a diversity of interesting properties for fundamental research and further applications $[1,2]$. Table 1 classifies $2 \mathrm{D}$ TMDs into semiconductors, metals and semimetals, according to their band structures. Especially for those semiconducting ones, their bandgaps are also listed. Moreover, owing to the special physical properties, metastable phases of TMDs have gained much attention. For instance, the $1 \mathrm{~T}-\mathrm{MoS}_{2}$ possesses superconductivity, magnetism, ferroelectricity and memristive behavior [34], which are potential for electronics, catalysis and energy storage. To obtain those metastable phases or construct the polymorphic heterostructures, giant efforts have been devoted to phase engineering of $2 \mathrm{D}$ TMDs, including phase transition and phase-selective synthesis.

\section{STRATEGIES OF PHASE TRANSITION FOR 2D TMDs}

The physical and chemical properties of $2 \mathrm{D}$ materials are dominated by their structures [70,71], and subtle structural changes could generate different properties [72-76]. Although the synthesis of 2D TMDs with stable phases has been mature, the direct fabrication of their metastable phases remains challenging. As a typical route, the phase transition is regarded as an efficient approach to obtain these metastable phases. Here, various phase transition strategies as well as their mechanisms are expounded.

\section{Intercalation}

Intercalation is one of the most common methods for the phase transition of 2D TMDs. Early in 1983, Haering et al. [70] reported that $\mathrm{Li}$ intercalation in $2 \mathrm{H}-\mathrm{MoS}_{2}$ induced a structural transformation, in which Mo coordination transformed from $2 \mathrm{H}$ to $1 \mathrm{~T}$ phase. Different from a rapid increase of the electron energy of $\mathrm{MoS}_{2}$ from $2 \mathrm{H}-\mathrm{MoS}_{2}$ to $2 \mathrm{H}-\mathrm{LiMoS}_{2}$ [77], the phase transition is attributed to the small increase in $1 \mathrm{~T}-\mathrm{LiMoS}_{2}$ without an energy gap. Besides, the transformation from $2 \mathrm{H}-\mathrm{MoSe}_{2}$ to $1 \mathrm{~T}-\mathrm{LiMoSe}_{2}$ can also be realized [70]. It is noteworthy that Li-intercalation is generally driven by external assistance, such as ball milling [78], electrochemical process $[79,80]$, and sonication $[81,82]$, which can help to
Table 1 Electrical properties of 2D TMDs of different phases

\begin{tabular}{|c|c|c|c|}
\hline TMD & Phase & Electronic characteristics & Ref. \\
\hline $\mathrm{TiS}_{2}$ & $1 \mathrm{~T}$ & Metallicity & [35] \\
\hline $\mathrm{TiSe}_{2}$ & $1 \mathrm{~T}$ & Metallicity & {$[36]$} \\
\hline $\mathrm{TiTe}_{2}$ & $1 \mathrm{~T}$ & Metallicity & [37] \\
\hline $\mathrm{ZrS}_{2}$ & $1 \mathrm{~T}$ & Semiconducting $(1.4 \mathrm{eV})$ & {$[38]$} \\
\hline $\mathrm{ZrSe}_{2}$ & $1 \mathrm{~T}$ & Semiconducting $(0.95 \mathrm{eV})$ & [39] \\
\hline $\mathrm{ZrTe}_{2}$ & $1 \mathrm{~T}$ & Dirac semimetallicity & [40] \\
\hline $\mathrm{HfS}_{2}$ & $1 \mathrm{~T}$ & Semiconducting $(1.45 \mathrm{eV})$ & [41] \\
\hline $\mathrm{HfSe}_{2}$ & $1 \mathrm{~T}$ & Semiconducting $(1.13 \mathrm{eV})$ & [39] \\
\hline $\mathrm{HfTe}_{2}$ & $1 \mathrm{~T}$ & Semimetallicity & {$[42]$} \\
\hline $\mathrm{VS}_{2}$ & $1 \mathrm{~T}$ & $\begin{array}{c}\text { Semiconducting ( } 0.3 \mathrm{eV}, 2-3 \text { layers) } \\
\text { Metallicity ( }>8 \text { layers })\end{array}$ & $\begin{array}{l}{[43]} \\
{[44]}\end{array}$ \\
\hline $\mathrm{VSe}_{2}$ & $1 \mathrm{~T}$ & Metallicity & [18] \\
\hline $\mathrm{VTe}_{2}$ & $1 \mathrm{~T}$ & Metallicity & {$[45]$} \\
\hline \multirow[t]{2}{*}{$\mathrm{NbS}_{2}$} & $2 \mathrm{H}$ & Metallicity & {$[46]$} \\
\hline & $1 \mathrm{~T}$ & Metallicity & [47] \\
\hline \multirow[t]{2}{*}{$\mathrm{NbSe}_{2}$} & $2 \mathrm{H}$ & Metallicity & {$[16]$} \\
\hline & $1 \mathrm{~T}$ & Metallicity & {$[48]$} \\
\hline $\mathrm{NbTe}_{2}$ & $1 \mathrm{~T}$ & Metallicity & {$[45]$} \\
\hline \multirow[t]{2}{*}{$\mathrm{TaS}_{2}$} & $2 \mathrm{H}$ & Metallicity & {$[49]$} \\
\hline & $1 \mathrm{~T}$ & Metallicity & {$[50]$} \\
\hline \multirow[t]{2}{*}{$\mathrm{TaSe}_{2}$} & $2 \mathrm{H}$ & Metallicity & {$[51]$} \\
\hline & $1 \mathrm{~T}$ & Metallicity & {$[52]$} \\
\hline $\mathrm{TaTe}_{2}$ & $1 \mathrm{~T}^{\prime}$ & Metallicity & {$[45]$} \\
\hline \multirow[t]{2}{*}{$\mathrm{MoS}_{2}$} & $2 \mathrm{H}$ & Semiconducting $(1.85 \mathrm{eV})$ & {$[53]$} \\
\hline & $1 \mathrm{~T}$ & Metallicity & {$[54]$} \\
\hline \multirow[t]{2}{*}{$\mathrm{MoSe}_{2}$} & $2 \mathrm{H}$ & Semiconducting $(1.55 \mathrm{eV})$ & {$[55]$} \\
\hline & $1 \mathrm{~T}$ & Metallicity & {$[56]$} \\
\hline \multirow[t]{3}{*}{$\mathrm{MoTe}_{2}$} & $2 \mathrm{H}$ & Semiconducting $(1.1 \mathrm{eV})$ & {$[57]$} \\
\hline & $1 \mathrm{~T}^{\prime}$ & Metallicity & {$[58]$} \\
\hline & $\mathrm{T}_{\mathrm{d}}$ & Weyl semimetallicity & {$[59]$} \\
\hline \multirow[t]{2}{*}{$\mathrm{WS}_{2}$} & $2 \mathrm{H}$ & Semiconducting $(2.02 \mathrm{eV})$ & {$[60]$} \\
\hline & $1 \mathrm{~T}$ & Metallicity & {$[25]$} \\
\hline \multirow[t]{2}{*}{$\mathrm{WSe}_{2}$} & $2 \mathrm{H}$ & Semiconducting $(1.7 \mathrm{eV})$ & {$[61]$} \\
\hline & $1 \mathrm{~T}$ & Metallicity & {$[62]$} \\
\hline \multirow[t]{2}{*}{$\mathrm{WTe}_{2}$} & $1 \mathrm{~T}^{\prime}$ & Semimetallicity & {$[63]$} \\
\hline & $\mathrm{T}_{\mathrm{d}}$ & Weyl semimetallicity & {$[64]$} \\
\hline $\mathrm{ReS}_{2}$ & $1 \mathrm{~T}^{\prime \prime}$ & Semiconducting $(1.43 \mathrm{eV})$ & {$[65]$} \\
\hline $\mathrm{ReSe}_{2}$ & $1 \mathrm{~T}^{\prime \prime}$ & Semiconducting $(1.22 \mathrm{eV})$ & {$[66]$} \\
\hline $\mathrm{PtS}_{2}$ & $1 \mathrm{~T}$ & Semiconducting $(1.75 \mathrm{eV})$ & {$[67]$} \\
\hline $\mathrm{PtSe}_{2}$ & $1 \mathrm{~T}$ & Semiconducting $(1.13 \mathrm{eV})$ & {$[68]$} \\
\hline $\mathrm{PtTe}_{2}$ & $1 \mathrm{~T}$ & Dirac semimetallicity & [69] \\
\hline
\end{tabular}


overcome the energy barrier of phase transition. Detailed phase transition mechanism and the intercalation stability have been widely studied. In general, $\mathrm{H}-\mathrm{MoS}_{2}$ changes into T-LiMoS 2 during the early lithiation on account of the unusual interaction of intrinsic doping and electronphonon coupling. In case of deep lithiation, $\mathrm{T}-\mathrm{LiMoS}_{2}$ would be transformed into $\mathrm{Li}_{2} \mathrm{~S}$ and Mo clusters [83]. In a word, the lithiation process should be controlled, otherwise the materials may be degraded. Besides the lithiation process, the environmental factors have impacts on the stability of Li-intercalated TMDs. To avoid such unexpected reversion from the as-obtained $1 \mathrm{~T}$ to the $2 \mathrm{H}$ phase [84-87], a thermal activated hydrogenation method is put forward to substitute $\mathrm{LiH}$ for $\mathrm{Li}$ [88]. Furthermore, the phase transition induced by intercalation also depends on the thickness of $\mathrm{MoS}_{2}$, because the layer number affects both the energy difference between $2 \mathrm{H}$ and $1 \mathrm{~T}^{\prime}-\mathrm{MoS}_{2}$ and the critical injected electron concentrations [89].

In situ characterization and theoretical calculation uncover the detailed processes. Bai et al. [90] studied the mechanism of dynamic electrochemical lithiated $\mathrm{MoS}_{2}$ processes by in situ high-resolution transmission electron microscopy (HRTEM). The phase transition of $\mathrm{MoS}_{2}$ is confirmed according to the polytype superlattice with a $\mathrm{Li}$ ion occupying the interlayer $\mathrm{S}-\mathrm{S}$ tetrahedron site. During the $\mathrm{Li}$ intercalation, the electron transfer from the intercalant to TMDs leads to the electron density increase of the $\mathrm{d}$ orbital of the transition metals, which induces the destabilization of the $2 \mathrm{H}$ phase and accelerates the phase transition to the metallic 1T phase. Jena et al. [91] generalized the probable pathways for the phase transition of $\mathrm{MoS}_{2}$ (Fig. 3a). At first, the trigonal prismatic $\mathrm{H}-\mathrm{MoS}_{2}$ starts to change into the octahedral coordinated $\mathrm{O}-\mathrm{MoS}_{2}$ (as the 1T phase mentioned above) when $\mathrm{Li}$ concentration reaches $20 \%$. Then, as the concentration of $\mathrm{Li}$ increases to $100 \%, \mathrm{O}-\mathrm{MoS}_{2}$ becomes more stable, and the atomic configuration converts from zigzag chains to "diamond chains" to ultimately form the stable DT- $\mathrm{MoS}_{2}$ (as the aforementioned 1T" phase). Next, DT- $\mathrm{MoS}_{2}$ turns into ZT- $-\mathrm{MoS}_{2}$ (equivalent to the $1 \mathrm{~T}^{\prime}$ phase), when all $\mathrm{Li}$ atoms are removed from the system. Finally, ZT- $\mathrm{MoS}_{2}$ converts back to the original $\mathrm{H}-\mathrm{MoS}_{2}$ by heating or aging. The pathways contribute to further understanding of the phase transition via Li intercalation.

Other intercalants including $\mathrm{Na}, \mathrm{K}$, and ammonium ions have been employed to achieve the TMD phase transition [92-95]. It is demonstrated that appropriate intercalant concentration results in the phase transition, whereas the excessive concentration would lead to the structural degradation [92]. Distinct from alkali metal intercalants, the intercalated ammonium ions can stabilize the $1 \mathrm{~T}-\mathrm{MoS}_{2}$ and $1 \mathrm{~T}-\mathrm{WS}_{2}$ [94,95], which may innovate the phase transition conventionally triggered by alkali metal intercalation.

In summary, intercalation is a general means of the phase transition of 2D TMDs, whose stability may rely on the intercalation extent or the environment. Additionally, more efficient and specific intercalation methods still remain to be explored.

\section{Charge transfer}

In consideration of the different splitting conditions of $d$ orbitals in different phases of 2D TMDs, charge transfer which tends to take place in the external electrical field or in the interfaces between materials is utilized to change the $\mathrm{d}$ orbital arrangements and induce the phase transition of 2D TMDs [21]. Promisingly, charge transfer can be nondestructive for the phase transition of $2 \mathrm{D}$ TMDs.

\section{Electrostatic doping}

Electrostatic doping is recognized as a damage-free strategy, which refers to the charge injection from an external electrostatic field. As theoretically predicted, the phase stability can be directly affected by external charge transfer [96], which is ascribed to the total energy difference of TMD phases associated with the charge density. Therefore, it is feasible to achieve the TMD phase transition by using the electrostatic gating devices. Reed et al. [96] discovered the imposed gate voltages serving as promoters through simulation. They demonstrated that the alteration of electronic chemical potential or carrier density can induce the phase transition of monolayer TMDs. On the basis of the prediction, Zhang et al. [97] achieved reversible phase transition between $2 \mathrm{H}$ and $1 \mathrm{~T}^{\prime}$ phases of monolayer $\mathrm{MoTe}_{2}$ by imposing and withdrawing the electrostatic doping in the gating device (Fig. 3b). To confirm the components of the $1 \mathrm{~T}^{\prime}$ and $2 \mathrm{H}$ phases, they fitted the relative Raman mixture with Lorentz model. The relative content of the two phases was expressed by correlation Raman peak intensity ratio. The hysteretic loop of the gate-dependent Raman intensity ratios (Fig. 3c) indicates the reversible phase transition as the continuously decrease and increase of the gate voltage. In detail, Raman intensity ratio, $F=\mathrm{A}_{\mathrm{g}}\left(1 \mathrm{~T}^{\prime}\right) /$ $\left(\mathrm{A}_{1}{ }^{\prime}(2 \mathrm{H})+\mathrm{A}_{\mathrm{g}}\left(1 \mathrm{~T}^{\prime}\right)\right)$, reflects the ratio of the phase transition, where $A_{g}\left(1 T^{\prime}\right)$ and $A_{1}{ }^{\prime}(2 H)$ refer to the Raman intensity of $\mathrm{A}_{\mathrm{g}}$ mode of $1 \mathrm{~T}^{\prime}-\mathrm{MoTe}_{2}$ and $\mathrm{A}_{1}{ }^{\prime}$ mode of the $2 \mathrm{H}-\mathrm{MoTe}_{2}$, respectively. Along the forward sweeping, the 

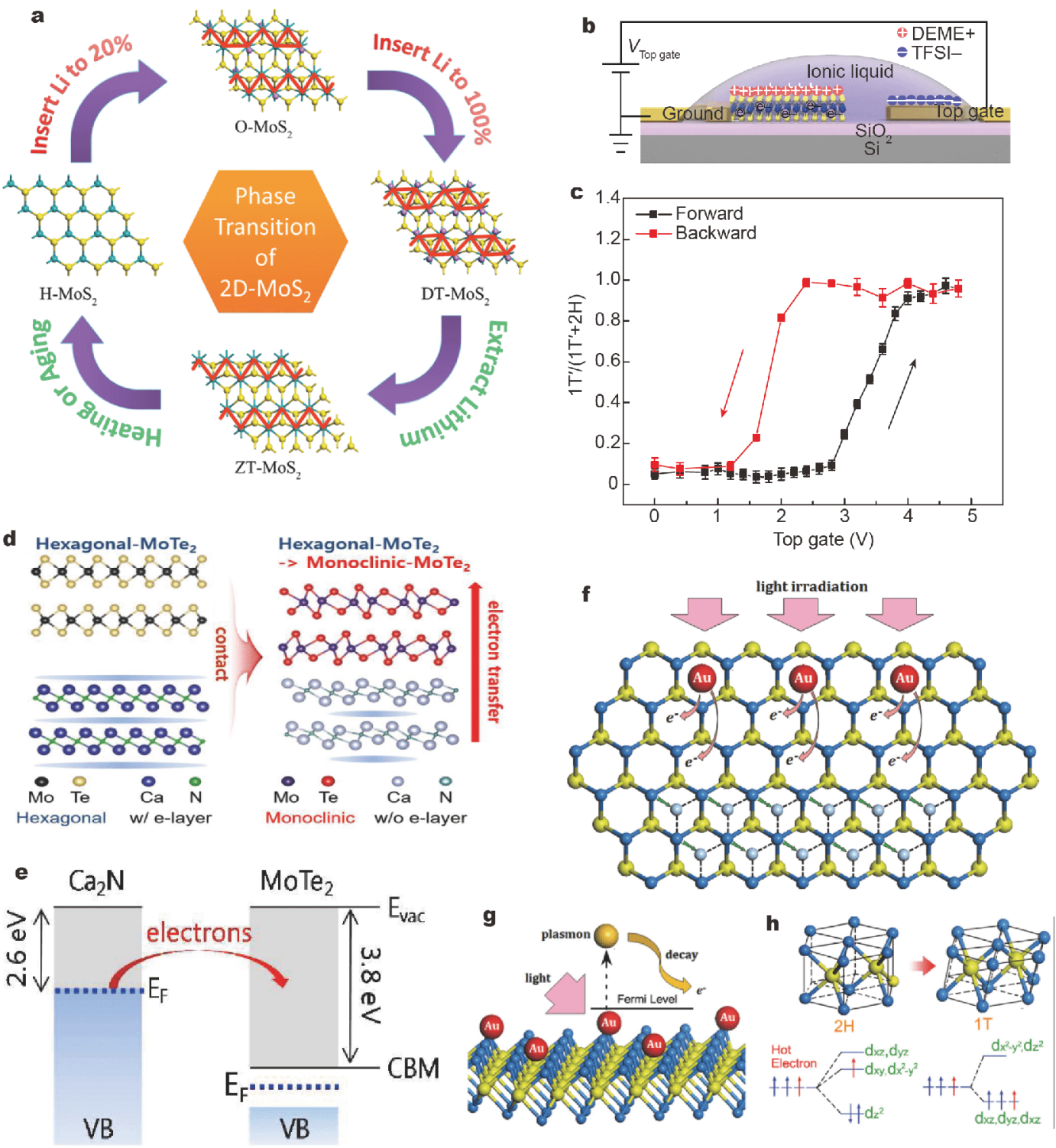

Figure 3 The phase transition of TMDs achieved by intercalation and charge transfer. (a) Possible paths of structural phase changes as lithium is intercalated in or extracted from $\mathrm{MoS}_{2}$ monolayer. Reproduced with permission from Ref. [91]. Copyright 2014, American Chemical Society. (b) Schematic illustration of the phase transition of exfoliated monolayer $\mathrm{MoTe}_{2}$ driven by electrostatic doping in a gating device. (c) Gate-dependent Raman intensity ratios $F$ ( $y$ axis) of $\mathrm{MoTe}_{2}$, showing a hysteresis loop with $1.8 \mathrm{~V}$ width under an electrical field scan. The red and black curves show decreasing and increasing gate voltage, respectively. Reproduced with permission from Ref. [97]. Copyright 2017, Springer Nature. (d) Schematic illustration of the phase transition of $\mathrm{MoTe}_{2}$ via interface electron transfer, when it is vertically contacted with $\left[\mathrm{Ca}_{2} \mathrm{~N}^{+} \cdot \mathrm{e}^{-}\right.$. (e) Band alignment of $\mathrm{MoTe}_{2}$ and $\left[\mathrm{Ca}_{2} \mathrm{~N}\right]^{+} \cdot \mathrm{e}^{-}$, indication the electron transfer in the interface. Reproduced with permission from Ref. [98]. Copyright 2017, American Chemical Society. (f-h) Schematic illustrations of plasmonic hot electron generated from Au induced phase transition of $\mathrm{MoS}_{2}$ from $2 \mathrm{H}$ to $1 \mathrm{~T}$ phase. Reproduced with permission from Ref. [99]. Copyright 2014, Wiley.

phase transition from $2 \mathrm{H}$ to $1 \mathrm{~T}^{\prime}$ phase begins at the gate voltage of $2.8 \mathrm{~V}$, and it reaches the complete transformation at $3.8 \mathrm{~V}$. When the gate voltage is swept backwards, the $2 \mathrm{H}$ phase starts to reappear at the gate voltage of $2.4 \mathrm{~V}$ and recovers completely at $1.2 \mathrm{~V}$. Moreover, the phase transition occurs uniformly across the entire monolayer without obvious morphologic changes. Electrostatic doping can provide a versatile platform to study new topological phases. Dynamically controlled phase transition in 2D TMDs exhibits promising prospects in phase- 
change electronics.

\section{Interface electron transfer}

In addition to charge injection via electrostatic doping, interface electron transfer also enables the phase transition by virtue of the charge transfer of different band alignments in contacting materials. Some materials, such as electrides and metals, take full advantage of free electrons to achieve the phase transition. Kim et al. [98] reported that the phase transition was triggered by the charge transfer, when $\mathrm{MoTe}_{2}$ was contacted with the single-crystalline $2 \mathrm{D}\left[\mathrm{Ca}_{2} \mathrm{~N}\right]^{+} \cdot e^{-}$owning a low work function of $\sim 2.6 \mathrm{eV}$ (Fig. 3d). The first-principles calculations show that the interface electron transfer is driven by the energy difference $(\sim 1.2 \mathrm{eV})$ between the conduction band minimum (CBM) of $2 \mathrm{H}-\mathrm{MoTe}_{2}$ and the Fermi level of $\left[\mathrm{Ca}_{2} \mathrm{~N}\right]^{+} \cdot e^{-}$, as shown in Fig. 3e. It is simulated that a strong electron doping happens after the contact of two materials, where the Fermi level of $\mathrm{MoTe}_{2}$ shifts $0.15 \mathrm{eV}$ above the CBM. The strategy may be potential for constructing semiconductor-metal junctions toward high-performance electronics. It is speculated that suitable electron donors contacting with TMDs can induce the phase transition. Fang et al. [99] demonstrated that hot electrons produced by plasmon excitation in $\mathrm{Au}$ nanospheres (Fig. 3f) are able to induce a transient reversible phase transition (Fig. 3g). The hot electrons can be effectively transferred to $\mathrm{MoS}_{2}$ monolayer, because of the low Schottky barrier $(0.8 \mathrm{eV})$ between $\mathrm{Au}$ and $\mathrm{MoS}_{2}$. In terms of crystal field theory, the Mo $4 \mathrm{~d}$ orbitals split into three energy levels in $2 \mathrm{H}-\mathrm{MoS}_{2}$ or two levels in $1 \mathrm{~T}$ $\mathrm{MoS}_{2}$, respectively [100,101]. Different from the unoccupied orbitals lying in the high-energy levels in $2 \mathrm{H}$ $\mathrm{MoS}_{2}$, there is an unoccupied orbital in the low-energy level in $1 \mathrm{~T}-\mathrm{MoS}_{2}$, in which the hot electrons tend to fill to stabilize the 1T phase (Fig. 3h). Meanwhile, deposited Au can also result in a local slide of top S plane due to the interaction between $\mathrm{MoS}_{2}$ and $\mathrm{Au}$, which can weaken the bonding of top Mo-S and further lead to a destabilization of the structure to promote the phase transition $[99,102]$.

To sum up, charge transfer, including electrostatic doping and interface electron transfer, plays an important role in the phase transition of TMDs for its damage-free process. Additionally, atomic doping can modulate the DOS of TMDs to induce the charge transfer, so it may be used in the phase transition of TMDs. Nb-doping can induce structural transition from $2 \mathrm{H}$ to $3 \mathrm{R}$ stacking in $\mathrm{MoS}_{2}$ due to the lower total energy of Nb-doped 3R than that of $\mathrm{Nb}$-doped $2 \mathrm{H}$, which can be ascribed to freecarrier screening of holes residing in the $\mathrm{d}_{z^{2}}$ bands of Nb- doped $\mathrm{MoS}_{2}$ [103,104]. Nevertheless, in consideration of the transformation of intrinsic properties, atomic doping is not very appropriate for the nondestructive phase transition.

\section{External irradiation}

External irradiation offers high-energy particles for the phase transition, including plasma [99,105], electron beam [102] and laser [106]. Here we review the detailed operation and the corresponding mechanism of the external irradiation strategies.

\section{Plasma}

Plasma treatment is a clean and scalable approach to phase transition owing to the high kinetic energy inducing the lateral sliding. However, it has also been reported that some vacancies are likely to occur during the plasma treating process $[107,108]$. Zhu et al. [105] reported a new phase transition way of Ar-plasma treatment being used to activate the $2 \mathrm{H} \rightarrow 1 \mathrm{~T}$ phase transition. As shown in Fig. 4a, an inductively coupled plasma generated by dispersing a $20 \mathrm{~W}$ radio frequency (RF) power at the entrance of a quartz tube furnace using an RF (13.56 MHz) coil is applied to the as-grown $\mathrm{MoS}_{2}$. It has been calculated that the kinetic energy of Ar ions is tuned well below the level where etching could occur but is sufficient to wrench the S-Mo bond to induce the lateral sliding of top S-layer. As shown in Fig. 4b, after inducing the phase transition, there are three weak but distinct new peaks $J_{1}\left(167 \mathrm{~cm}^{-1}\right), J_{2}\left(227 \mathrm{~cm}^{-1}\right)$ and $J_{3}$ $\left(334 \mathrm{~cm}^{-1}\right)$ appearing, which is in good agreement with the signature of the monolayer 1T- $\mathrm{MoS}_{2}$ (inset of Fig. 4b). To sum up, plasma treating can open up a novel route for phase engineering in 2D TMDs, but it still needs further development in the controllability.

\section{Electron beam}

Electron-beam irradiation is an efficient method to achieve the phase transition, ascribing to high-energyinduced lattice reconstruction. Lin et al. [102] introduced the transformation from $2 \mathrm{H}$ to $1 \mathrm{~T}$ of a single layer of $\mathrm{MoS}_{2}$ under electron-beam irradiation. The $2 \mathrm{H}$ phase converts into a new phase by forming two band-like structures with an angle of $60^{\circ}$. Each stripe consists of three to four constricted $\mathrm{MoS}_{2}$ zigzag chains ( $\alpha$ in Fig. 4d) with decreased distance between two Mo atoms, at the intersection of which the over packed atoms trigger the formation of $1 \mathrm{~T}$ phase to release the stress. With continuous electron-beam scanning, the $1 \mathrm{~T}$ phase domain progressively forms a triangular shape, and at 

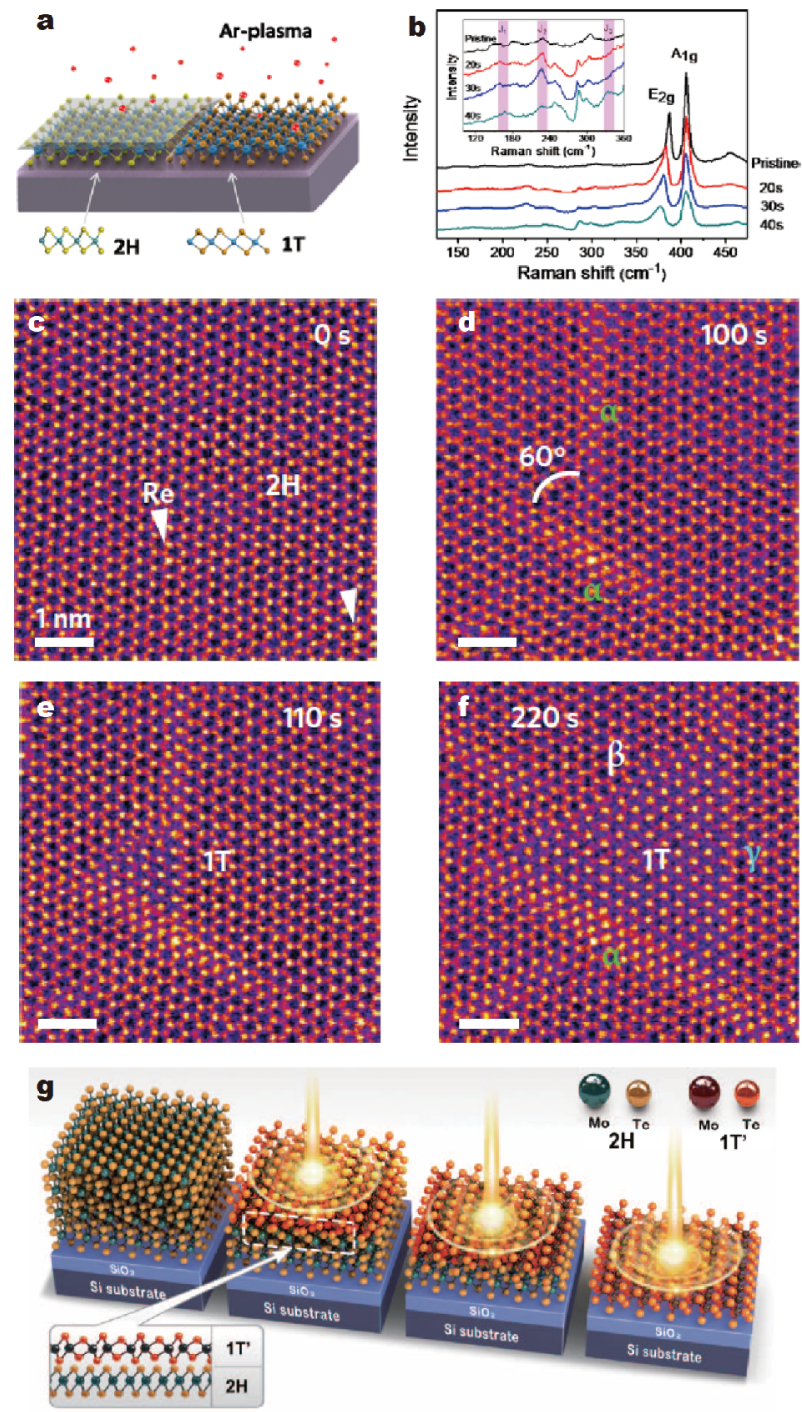

Figure 4 Phase transition induced by external irradiation. (a) Schematic representation of the plasma treatment process. (b) Time-dependent Raman spectra of monolayer $\mathrm{MoS}_{2}$ and plasma-treated $\mathrm{MoS}_{2}$. Reproduced with permission from Ref. [105]. Copyright 2017, American Chemical Society. (c-f) Atomic movements during $2 \mathrm{H} \rightarrow 1 \mathrm{~T}$ phase transformation in the monolayer $\mathrm{MoS}_{2}$ under electron beam irradiation. Reproduced with permission from Ref. [102]. Copyright 2014, Springer Nature. (g) Schematic representation of the laser-irradiation process. Reproduced with permission from Ref. [106]. Copyright 2015, the American Association for the Advancement of Science.

the same time as two new phase boundaries denoted as $\beta$ and $\gamma$ show two new atomic arrangements (Fig. 4c-f). Thus, the electron beam can be applied to intentionally induce the phase transition in a target area with a predetermined size toward specific nanoelectronics.

\section{Laser}

Laser irradiation, regarded as the high-energy photon beam, can achieve the phase transition without unexpected doping impurities. Cho et al. [106] designed a kind of local polymorph engineering, that is, they drove the phase transition from the $2 \mathrm{H}$ to $1 \mathrm{~T}^{\prime}$ phase in $\mathrm{MoTe}_{2}$ via laser irradiation in a selected area. The researchers have confirmed that the top layer initially experienced the phase transition with the thinning effect, as shown in Fig. $4 \mathrm{~g}$, whereas the reverse phase transition was not observed by laser irradiating even with a higher energy or intensity of the laser.

Generally, these three techniques are facile to realize the controlled phase transition, due to the controllability of plasma, electron beam and laser. Nevertheless, high energy particles tend to bring some damage to the sample. Moreover, it is also worth mentioning that external irradiation may offer the patternable phase transition in the future, which is promising to be extended to industrial applications.

\section{Thermal treatment}

Thermal treatment is identified as one of the most convenient strategies, for its excellent controllability of the phase transition at different temperatures according to phase diagrams. The phase transition can easily occur with the frequent vibrations of atoms and the formations of new chemical bonds at a high temperature. Lee et al. [109] reported the synthesis of $2 \mathrm{H}$ - and $1 \mathrm{~T}^{\prime}-\mathrm{MoTe}_{2}$ in a two-zone chemical vapor deposition (CVD) system. At first, they obtained large-scale $2 \mathrm{H}-\mathrm{MoTe}_{2}$ via slow tellurization methods. In Fig. 5a, during the slow tellurization, the $1 \mathrm{~T}^{\prime}-\mathrm{MoTe}_{2}$ initially took place in the top region followed by the gradual transformation into $2 \mathrm{H}-\mathrm{MoTe}_{2}$. The researchers also presented that the reverse phase transition could occur by further tellurization or annealing rapidly. Kim et al. [110] obtained $2 \mathrm{H}$ - and $1 \mathrm{~T}^{\prime}-\mathrm{MoTe}_{2}$ via the flux method. As the phase diagram shown in Fig. 5b, the engineering of single crystalline $2 \mathrm{H}-$ and $1 \mathrm{~T}^{\prime}-\mathrm{MoTe}_{2}$ can be realized. In the phase diagram (Fig. $5 \mathrm{~b}$ left), the $2 \mathrm{H}$ phase starts to convert into the stable $1 \mathrm{~T}^{\prime \prime}$ phase at temperatures higher than $500^{\circ} \mathrm{C}$ (Fig. $5 \mathrm{~b}$ right). Besides, the $2 \mathrm{H}$ phase can recover as it slowly cools with a new mixed phase appearing in a temperature range between 500 and $820^{\circ} \mathrm{C}$, which illustrates the reversible structural phase transition. $2 \mathrm{H}$ phase can be obtained by slow cooling from $900^{\circ} \mathrm{C}$ to room temperature, while $1 \mathrm{~T}^{\prime}$ phase can be synthesized by quenching or rapid cooling process. In situ variabletemperature X-ray diffraction (XRD) patterns are 

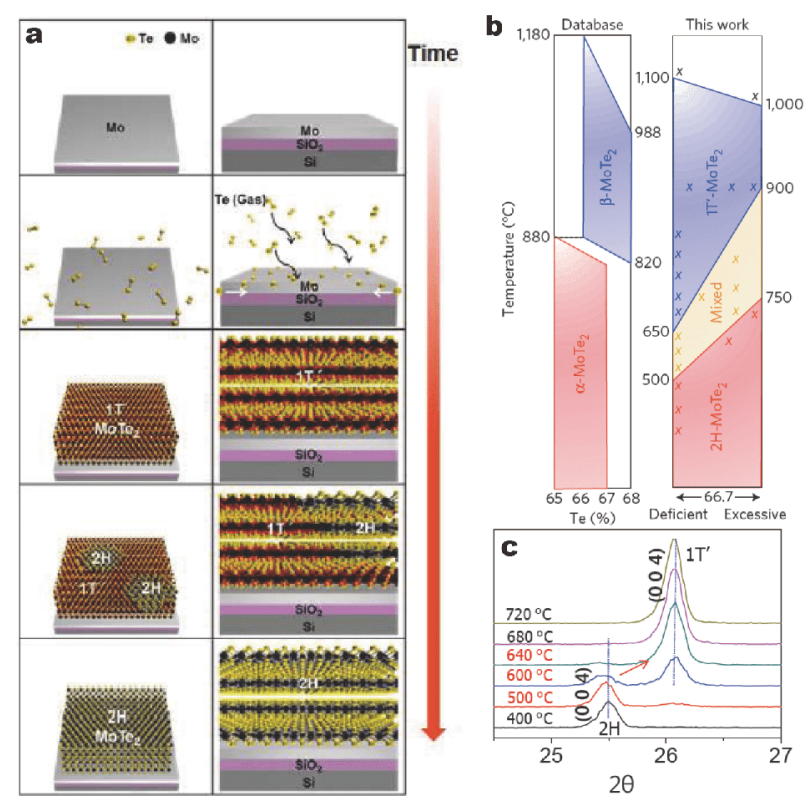

Figure 5 Phase transition induced by thermal treatment. (a) Schematic illustrations of the growth of $2 \mathrm{H}-\mathrm{MoTe}_{2}$ and the phase transition from $2 \mathrm{H}$ to $1 \mathrm{~T}^{\prime}$ phase. Reproduced with permission from Ref. [109]. Copyright 2015, American Chemical Society. (b) Phase diagram of $\mathrm{MoTe}_{2}$. (c) In situ XRD results of $\mathrm{MoTe}_{2}$ at different thermal treatment temperatures. Reproduced with permission from Ref. [110]. Copyright 2015, Springer Nature.

presented in Fig. 5c, which also proves that the structural phase transition begins at $500^{\circ} \mathrm{C}$ until the $2 \mathrm{H}-\mathrm{MoTe}_{2}$ completely transforms into $1 \mathrm{~T}^{\prime}-\mathrm{MoTe}_{2}$ at $680^{\circ} \mathrm{C}$. Besides, it is also reported that group VB TMDs such as $\mathrm{TaS}_{2}$ can realize phase transition by this approach. Wang et al. [111] found the $1 \mathrm{~T} \rightarrow 2 \mathrm{H}$ polymorph transition on the surface of bulk- $\mathrm{TaS}_{2}$ by thermal annealing because of the thermally induced relaxation derived from larger freedom for atom displacement. Although thermal methods are very efficient and convenient, the biggest problem lies in the precise control of the conditions and transition process, which might cause many limits especially in the applications of the few-layer materials.

\section{Stress induction}

Stress induction, which utilizes mechanical force to change the lattice structure of TMDs, provides another viable strategy for the phase transition. It is mainly realized by two different methods: tensile strain and hydrostatic pressure. The former realizes the transition via interlayer atomic plane gliding, while the latter distorts the lattice structure mechanically. Duerloo et al. [33] demonstrated that an equibiaxial tensile strain of $10 \%-15 \%$ was needed to achieve the phase transition for most of TMDs, among which it was the easiest to achieve in the case of $\mathrm{MoTe}_{2}$ through a tensile strain less than 1.5\%. Experimentally, Lee et al. [112] proposed a method to enable a reversible phase transition for $\mathrm{MoTe}_{2}$ with a tensile strain exerted by an atomic force microscope (AFM) tip. As a function of forces, the system resistance changes by nearly four orders of magnitude, suggesting that $\mathrm{MoTe}_{2}$ experiences the phase transition from semiconducting $2 \mathrm{H}$ phase to metallic $1 \mathrm{~T}^{\prime}$ phase under the tensile strain. Fully reversible transformation occurs after the release of strain. According to the forcetemperature phase diagram in Fig. 6b, tensile strain can induce the phase transition at a certain temperature, and the needed strain becomes smaller as the temperature increases. The tensile strain may also induce the phase transition for other TMDs with underlying effects in addition to heat (e.g., electric fields and chemical doping).

It seems like a compressive stress to exert hydrostatic pressure on TMDs to induce the phase transition. Akinwande et al. [113] used a diamond anvil cell (DAC) to exert a uniform hydrostatic pressure on the $\mathrm{MoS}_{2}$. As shown in Fig. 6c, it is revealed by theoretical calculation that the bandgap of the $2 \mathrm{H}-\mathrm{MoS}_{2}$ monolayer first increases until it reaches the peak as the applied pressure increases. Above the critical pressure, the bandgap gradually reduces, and finally the metallization of $2 \mathrm{H}-\mathrm{MoS}_{2}$ is predicted to be achieved at about $68 \mathrm{GPa}$. In addition, it is found that interlayer interactions play a significant role in the phase transition. That is, pressure acquired to achieve transition decreases as the layer number increases (Fig. 6d).

Though tensile strain applied by AFM can achieve the phase transition, it holds a notable feature that a phase reversal will occur after strain release, and thus it is unrealistic to obtain stable $1 \mathrm{~T}^{\prime}$-TMDs by this method. Nevertheless, it is possible to be applied in the development of biological and optical sensors, on account of environmentally sensitive electrical property changes owing to the phase transition. Producing large hydrostatic pressure through DAC also faces the challenge to reach such high pressure, which hinders the practical application of the stress-induced phase transition.

\section{Others}

Besides intercalation, charge transfer, external irradiation, thermal treatment and stress induction, there are still many other methods of phase engineering. Xu et al. [73] utilized supercritical $\mathrm{CO}_{2}$ in the intermediate layer of the as-exfoliated $\mathrm{MoS}_{2}$ film to partially convert the $2 \mathrm{H}-\mathrm{MoS}_{2}$ nanosheets into the $1 \mathrm{~T}$ phase and form lattice-matched 

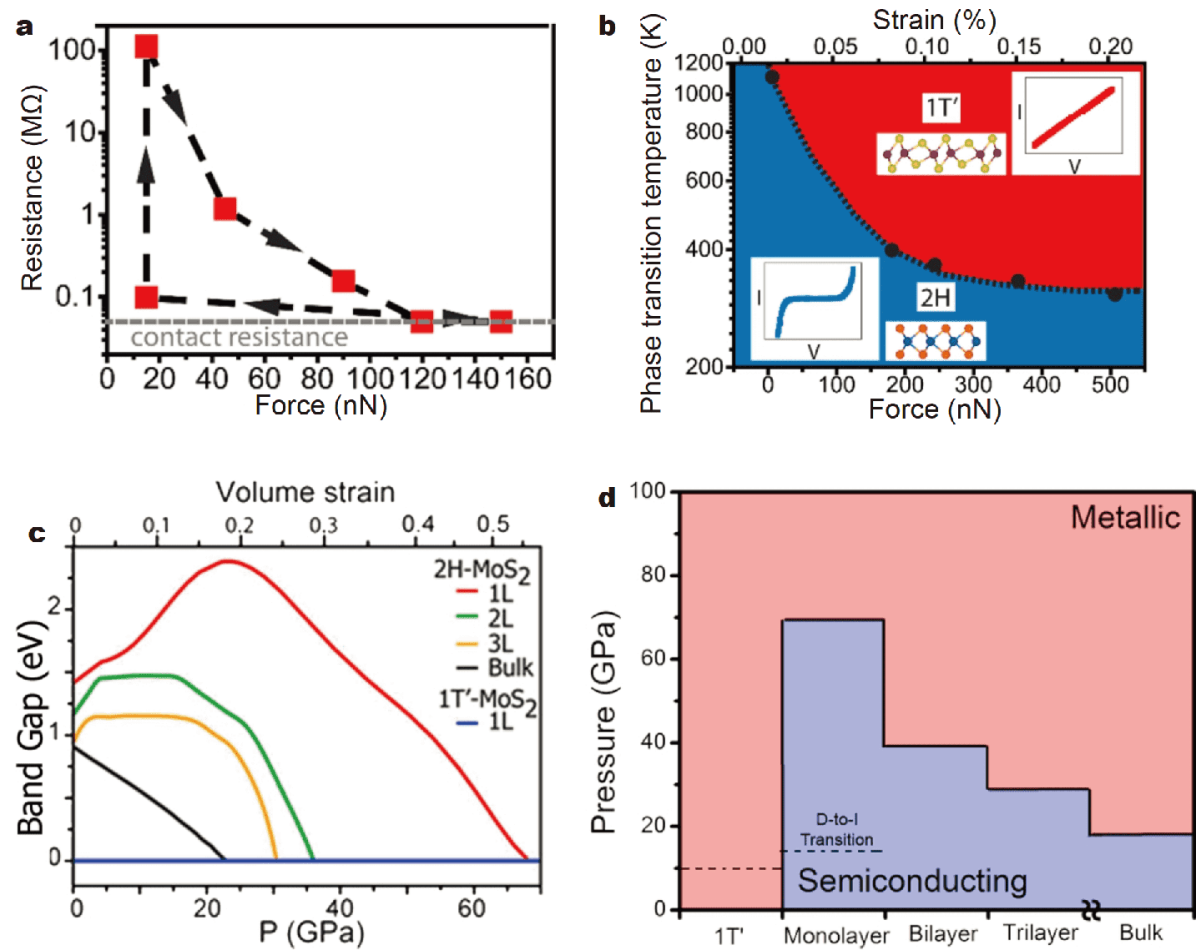

Figure 6 Phase transition achieved via stress induction. (a) Resistance plots of $\mathrm{MoTe}_{2}$ with different tensile forces applied. The arrows show the reversible metallization of $2 \mathrm{H}-\mathrm{MoTe}_{2}$. (b) Phase diagram as a function of phase transition temperature and the tensile force. Insets show the structures and electrical properties of different phase $\mathrm{MoTe}_{2}$. Reproduced with permission from Ref. [112]. Copyright 2016, American Chemical Society. (c) Bandgap changes as a function of hydrostatic pressure of $2 \mathrm{H}-$ and $1 \mathrm{~T}-\mathrm{MoS}_{2}$ with different layer numbers. (d) Electrical characteristics of $2 \mathrm{H}-$ and $1 \mathrm{~T}$ $\mathrm{MoS}_{2}$ with different layer numbers when the hydrostatic pressure changes. Reproduced with permission from Ref. [113]. Copyright 2015, American Chemical Society.

heterojunction. Moreover, the available conductivity of the $1 \mathrm{~T}$ phase and the extended life of photogenerated electrons make such tailored $\mathrm{MoS}_{2}$ perform high catalytic activity for water splitting. Besides, Chhowalla et al. [114] introduced the covalent functionalization of TMDs which relied on the electron transfer between the electron-rich metallic $1 \mathrm{~T}$ phase and the covalent functional groups of an organohalide reactant instead of defect engineering. The reactions between amide and methyl moieties (from organoiodide precursors) successfully applied to $\mathrm{MoS}_{2}$, $\mathrm{WS}_{2}$ and $\mathrm{MoSe}_{2}$ make the properties of the $1 \mathrm{~T}$ phase transform from metallic to semiconducting. These special methods make it possible to combine the phase transition with solution surroundings, which may be applied in catalysis and sensing. Besides, as for alloyed phase heterostructures, there are still some approaches to partial phase transformation. Huang et al. [115] successfully obtained alloyed $\mathrm{Mo}_{x} \mathrm{~W}_{1-x} \mathrm{~S}_{2}$ nanosheets by liquid-phase preparation and controlled the $1 \mathrm{~T}$ concentration by altering the reaction temperature. They finally observed that a mixed $1 \mathrm{~T} / 2 \mathrm{H}$ phase $\mathrm{Mo}_{x} \mathrm{~W}_{1-x} \mathrm{~S}_{2}$ with a $1 \mathrm{~T}$ concentration of $\sim 60 \%$ presented the best performance towards hydrogen evolution reaction (HER).

\section{PHASE-SELECTIVE SYNTHESIS OF 2D TMDs}

2D TMDs with different phases, especially for the metastable ones, can be effectively obtained through the aforementioned phase transition strategies, including intercalation, charge transfer, external irradiation, thermal treatment and stress induction. Despite the fact that the target phase of 2D TMDs can be efficiently and controllably gained via the phase transition, there are still some shortcomings. 1) The as-obtained phases may not keep stable after withdrawing the external factors for the phase transition, so they would be transformed reversibly to the original phases. 2) Atomic vacancies induced by high external energy for the phase transition would lead to the unexpected property modulation, as well as chemical doping originating from intercalation. Therefore, it is urgent to develop controllable fabrication strategies for high phase-purity TMDs. Recently, phase- 

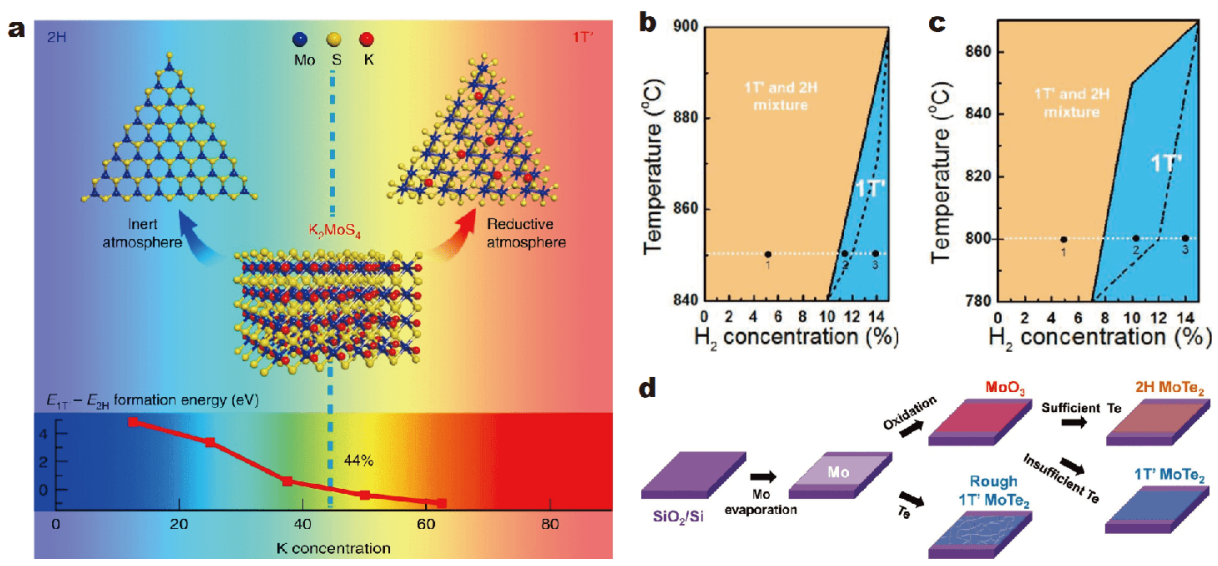

Figure 7 Phase-selective synthesis depending on precursor design. (a) Illustration of selective synthesis of $2 \mathrm{H}-$ or $1 \mathrm{~T}^{\prime}$ - $\mathrm{MoS}_{2}$ under different atmospheres with $\mathrm{K}_{2} \mathrm{MoS}_{4}$ precursor. The inset plots at the bottom show the formation-energy difference between $1 \mathrm{~T}^{\prime}-$ and $2 \mathrm{H}-\mathrm{K}_{x} \mathrm{MoS}_{2}$ as a function of $\mathrm{K}$ concentration. (b, c) Phase diagrams for the growth of $\mathrm{MoS}_{2}$ using $\mathrm{Rb}_{2} \mathrm{MoS}_{4}$ and $\mathrm{Cs}_{2} \mathrm{MoS}_{4}$ precursor, respectively. Reproduced with permission from Ref. [119]. Copyright 2018, Springer Nature. (d) Illustration of the phase-selective growth process for $2 \mathrm{H}$ - and $1 \mathrm{~T}^{\prime}-\mathrm{MoTe}_{2}$ by using $\mathrm{MoO}_{3}$ and $\mathrm{Mo}$ as precursors or tuning the Te flux with $\mathrm{MoO}_{3}$ serving as precursor. Reproduced with permission from Ref. [121]. Copyright 2016, John Wiley and Sons.

selective synthesis has been utilized to achieve high phase purity and construct polymorphic heterostructures.

\section{Precursor design}

Precursor design plays an important role in the TMDs growth, because the precursors affect the reaction thermodynamically. As a demonstration, the controllable and large-scale synthesis of $2 \mathrm{H}-\mathrm{MoS}_{2}$ has been achieved [116-118], but there are still some challenges existing in the fabrication of metastable $1 \mathrm{~T}\left(1 \mathrm{~T}^{\prime}\right)-\mathrm{MoS}_{2}$ [33]. To stabilize the $1 \mathrm{~T}^{\prime \prime}$ phase, Jiao et al. [119] prepared $1 \mathrm{~T}^{\prime}-\mathrm{MoS}_{2}$ by using $\mathrm{K}_{2} \mathrm{MoS}_{4}$ as Mo source. It is theoretically calculated that $1 \mathrm{~T}^{\prime}-\mathrm{K}_{x} \mathrm{MoS}_{2}$ holds higher stability than $2 \mathrm{H}-\mathrm{K}_{x} \mathrm{MoS}_{2}$ as the $\mathrm{K}$ concentration rises up to $44 \%$ (Fig. 7a). Other precursors, such as $\mathrm{Cs}_{2} \mathrm{MoS}_{4}$ and $\mathrm{Rb}_{2} \mathrm{MoS}_{4}$, can also be utilized to achieve the phaseselective synthesis of $1 \mathrm{~T}^{\prime \prime}-\mathrm{MoS}_{2}$ (Fig. $7 \mathrm{~b}$ and c). In addition, such a strategy is suitable to obtain $1 \mathrm{~T}^{\prime}-\mathrm{WS}_{2}$ [119] and $1 \mathrm{~T}^{\prime}-\mathrm{MoSe}_{2}$ [56]. Besides, precursor design can also be employed to the phase-selective synthesis of $\mathrm{MoTe}_{2}$. Kong et al. [120] reported that the component of the Mo source was essential for the CVD growth of $\mathrm{MoTe}_{2}$. The structure of $\mathrm{MoTe}_{2}$ strongly relies on the Mo oxidation state, as well as the tellurization efficiency. Therefore, the phase-selective synthesis of pure 1T'- and $2 \mathrm{H}-\mathrm{MoTe}_{2}$ in CVD is realized by using $\mathrm{Mo}$ and $\mathrm{MoO}_{3}$ as Mo precursors, respectively (Fig. 7d) [121]. Moreover, $1 \mathrm{~T}^{\prime}-\mathrm{MoTe}_{2}$ is predicted to be thermodynamically stable in the presence of certain mechanical stress [33]. In other words, when Mo foil serves as the precursor, a large stress may be generated during the formation of $1 \mathrm{~T}^{\prime}-\mathrm{MoTe}_{2}$, derived from the $380 \%$ increase of unit cell volume from Mo to $\mathrm{MoTe}_{2}$. While $\mathrm{MoO}_{3}$ acts as the precursor, the lower volume change ( 47\%) from $\mathrm{MoO}_{3}$ to $\mathrm{MoTe}_{2}$ can be released during the high-temperature growth, and then thermodynamically stable $2 \mathrm{H}-\mathrm{MoTe}_{2}$ without strain can be obtained. Furthermore, $\mathrm{Te}$ atomic flux has an extraordinary impact on the $\mathrm{MoTe}_{2}$ phases. With a sufficient amount of Te, $2 \mathrm{H}-\mathrm{MoTe}_{2}$ is supposed to be obtained whereas $1 \mathrm{~T}^{\prime}-\mathrm{MoTe}_{2}$ is obtained in the insufficient Te supply (Fig. 7d). In addition, special precursor supplement, such as Mo nanoislands deposited on substrates, can also be used for the phase-selective synthesis of $\mathrm{MoTe}_{2}$ [122].

In brief, there are two main strategies of precursor design to achieve the phase-selective synthesis of TMDs, including the metal precursor selection and the chalcogen flux regulation. Precursor design exerts a great influence on the synthesis at the aspect of both thermodynamics and dynamics, but it remains further understanding for the mechanism and the universality for group VB TMDs.

\section{Temperature control}

Temperature control is also an efficient approach to direct synthesis of expected TMD phases, which mainly relies on controlling the growth temperature. Different from the thermal treatment which achieves the target phase based on as-obtained samples, temperature control aims at directly synthesizing different phase by changing temperature without any post treatment. Furthermore, researchers prefer to determine the synthetic pathway based on some specific products with ground-state energy 


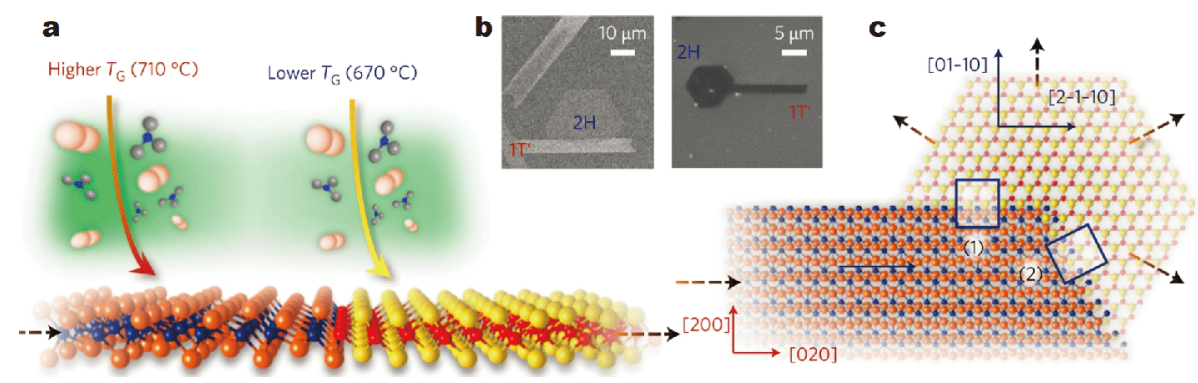

Figure 8 Phase-selective synthesis via temperature control. (a) Sequential growth of coplanar heteroepitaxy of $1 \mathrm{~T}^{\prime} / 2 \mathrm{H}^{-\mathrm{MoTe}_{2}}$ polymorphs. (b) SEM images of the $1 \mathrm{~T}^{\prime}-\mathrm{MoTe}_{2}-2 \mathrm{H}-\mathrm{MoTe}_{2}$ heterostructures. (c) Schematics of the heteroepitaxial structure. Reproduced with permission from Ref. [57]. Copyright 2017, Springer Nature.

differences. Here we summarize the temperature control strategies in phase-selective synthesis for single phase crystals and polymorphic heterostructures, including direct vapor growth and van der Waals epitaxy.

Direct vapor growth of 2D polymorphs with distinct phase selectivity is an efficient but elusive way probably due to thermodynamic instability. Jo et al. [57] applied such an approach in the synthesis of $\mathrm{MoTe}_{2}$ according to the relatively small difference in the ground-state energy between the $1 \mathrm{~T}^{\prime}$ and $2 \mathrm{H}$ phases. They used pure $\mathrm{MoO}_{3}$ and $\mathrm{Te}$ as precursors, with $\mathrm{NaCl}$ applied to selectively stabilize different phase. As shown in Fig. 8a-c, when the growth temperature $\left(T_{\mathrm{G}}\right)$ reaches $710^{\circ} \mathrm{C}$, the as-obtained rectangular- or trapezoidal-facet crystals are identified as $1 \mathrm{~T}^{\prime}-\mathrm{MoTe}_{2}$. At $T_{\mathrm{G}}=670^{\circ} \mathrm{C}$, it mainly forms hexagonal or triangular facets corresponding to $2 \mathrm{H}-\mathrm{MoTe}_{2}$. At intermediate $T_{\mathrm{G}}\left(670^{\circ} \mathrm{C}<T_{\mathrm{G}}<710^{\circ} \mathrm{C}\right)$, it tends to produce varied ratios of $1 \mathrm{~T}^{\prime} / 2 \mathrm{H}$ mixtures. The researchers successfully demonstrated heteroepitaxial integration of few-layer $\mathrm{MoTe}_{2}$ metal-semiconductor polymorphs within the same atomic planes which can be regarded as a promising atomic-scale electrical contact.

Van der Waals epitaxy is also a crucial way to grow various TMDs. Chen et al. [123] realized selective growth of specific phase $\mathrm{WSe}_{2}$ at different temperatures via such a method. It is illustrated that a single-layer $\mathrm{WSe}_{2}$ grown at a substrate temperature of $280^{\circ} \mathrm{C}$ exhibits a mixture of $1 \mathrm{H}$ and $1 \mathrm{~T}^{\prime}$ phases, and with the temperature climbing, the $1 \mathrm{H}$ phase plays a more dominant role until the $1 \mathrm{~T}^{\prime}$ phase totally disappears at growth temperature above $400^{\circ} \mathrm{C}$. What's more, the $1 \mathrm{~T}^{\prime}$ phase prefers to grow at a lower temperature and at the growth temperature of $130^{\circ} \mathrm{C}$ all the products convert into $1 \mathrm{~T}^{\prime \prime}$ phase. Monolayer $1 \mathrm{~T}^{\prime}-\mathrm{WSe}_{2}$ with a sizable quantum spin Hall (QSH) gap is conducive to further ambient-temperature spintronics, compared with $1 \mathrm{~T}^{\prime}-\mathrm{WTe}_{2}$ owning a smaller QSH gap. Besides, unlike exfoliated materials, the $1 \mathrm{~T}^{\prime}-\mathrm{WSe}_{2}$ film synthesized by molecular beam epitaxy (MBE) has a wider range of applications owing to the large-scale fabrication of devices such as topological field effect transistors (FETs).

In summary, phase-selective synthesis via temperature control is so facile and straightforward that attracts much attention. Just differing in practical steps, target phase TMDs, especially for metastable phases, can be obtained via the aforementioned methods, which offer a great platform for further studies and potential applications. In addition, taking temperature and percentages of precursors into account might make a difference.

\section{Atmosphere regulation}

Apart from the precursor design and temperature control, atmosphere also dynamically affect the phase-selective synthesis. As a reductive reagent, $\mathrm{H}_{2}$ plays an important role in the TMDs growth as a kinetic effect [4]. Jiao et al. [119] reported that $\mathrm{H}_{2}$ can accelerate the chemical transformation from $\mathrm{K}_{2} \mathrm{MoS}_{4}$ to $\mathrm{K}_{x} \mathrm{MoS}_{2}$. As shown in Fig. 9a, 1T'-2H mixed phase $\mathrm{MoS}_{2}$ can be transformed to pure $1 \mathrm{~T}^{\prime}$ phase as the $\mathrm{H}_{2}$ concentration ascends. Fig. 9b-d show the optical images of typical synthesis results corresponding to different $\mathrm{H}_{2}$ concentrations marked with $1-3$ in Fig. 9a. Owing to high $\mathrm{H}_{2}$ concentrationsx, aforementioned $\mathrm{K}$ concentration can be kept in a relatively high value and the $1 \mathrm{~T}^{\prime}$ phase $\mathrm{MoS}_{2}$ tends to be obtained. According to Fig. 9a, $\mathrm{H}_{2}$ concentration shows a kinetic effect based on the particular precursor design and temperature control. Nevertheless, the growth of metastable phase TMDs is not likely to only depend on atmosphere regulation, because the carrier gas can only help with the dynamic process rather than the thermodynamic process. For TMD growth, $\mathrm{H}_{2}$ is utilized to improve the reactivity of nonmetal precursors and regulate the expanding or etching along the TMD crystal edges. Therefore, atmosphere regulation can be an 

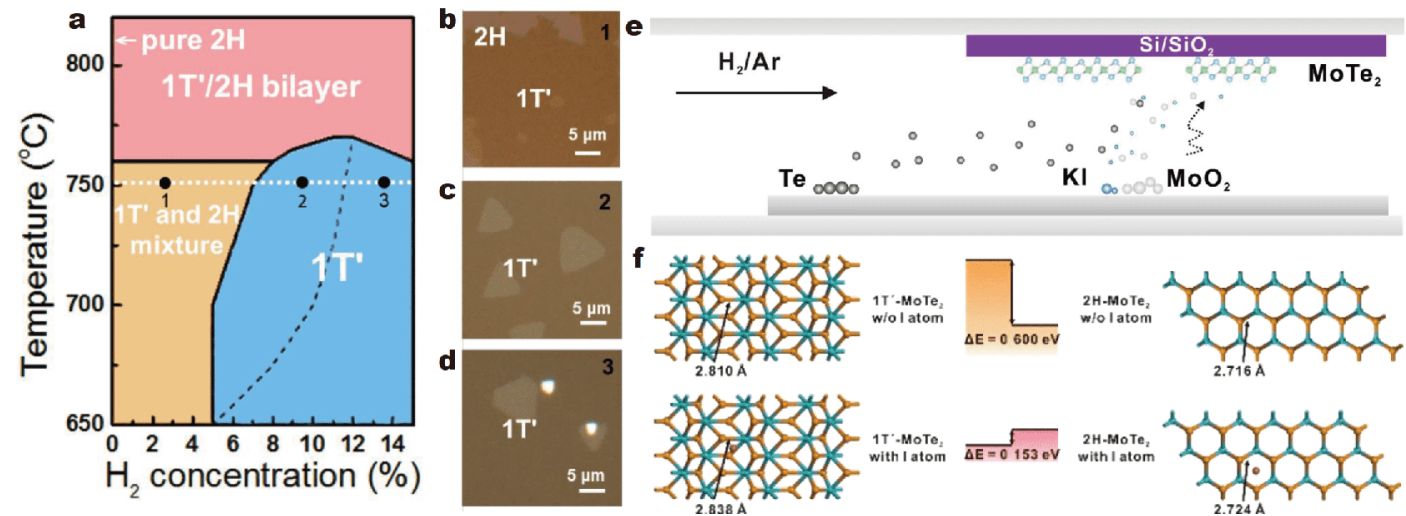

Figure 9 Atmosphere regulation and medium assistance for phase-selective synthesis. (a) Phase diagram of $1 \mathrm{~T}^{\prime}$ and $2 \mathrm{H}$ MoS 2 . (b-d) The optical images of growth results corresponding to different $\mathrm{H}_{2}$ concentrations marked with 1-3 in (a). Reproduced with permission from Ref. [119]. Copyright 2018, Springer Nature. (e) Schematic illustration of iodine-assisted 1T'-MoTe ${ }_{2}$ CVD growth. (f) Mechanism of the stabilizing of the 1T" phase and the bond parameters change by iodine assistance. Reproduced with permission from Ref. [58]. Copyright 2017, American Chemical Society.

indirect and assistant strategy to improve the phase selectivity.

\section{Medium assistance}

During the TMDs growth, the volatility of precursors and intermediates is crucial for the CVD process. Initially presented by Eda et al. [124] and then developed by Liu et al. [32], medium assistance has become one of the most effective ways to fabricate TMDs. Halide assistance aims at producing volatile intermediates and improving the reactivity. Xu et al. [125] reported millimeter-scale 1T'$\mathrm{MoTe}_{2}$ single crystals by using $\mathrm{KCl}$ and $\mathrm{NaCl}$ as growth promoters. The halides react with Mo precursor to produce volatile $\mathrm{MoO}_{2} \mathrm{Cl}_{2}$ and $\mathrm{MoOCl}_{4}$, and then these intermediates accelerate the reaction with $\mathrm{Te}$. However, it may not uncover the reason why the metastable $1 \mathrm{~T}^{\prime}$ phase was obtained rather than the stable $2 \mathrm{H}$ phase of $\mathrm{MoTe}_{2}$. $\mathrm{Fu}$ et al. [58] developed an iodine-assisted strategy to fabricate $1 \mathrm{~T}^{\prime}-\mathrm{MoTe}_{2}$. Fig. 9e shows the schematic illustration of the $1 \mathrm{~T}^{\prime}-\mathrm{MoTe}_{2}$ growth. KI is not only utilized to produce volatile $\mathrm{MoO}_{2} \mathrm{I}_{2}$, but also to stabilize the $1 \mathrm{~T}^{\prime}$ structure. As shown in Fig. 9f, the total energy of $1 \mathrm{~T}^{\prime}-\mathrm{MoTe}_{2}$ is calculated to be $0.600 \mathrm{eV}$ higher than that of $2 \mathrm{H}-\mathrm{MoTe}_{2}$, while the energy of iodine-adsorbed $1 \mathrm{~T}^{\prime}$ $\mathrm{MoTe}_{2}$ is $0.153 \mathrm{eV}$ lower than that of iodine-adsorbed $2 \mathrm{H}-\mathrm{MoTe}_{2}$. Furthermore, compared with $2 \mathrm{H}-\mathrm{MoTe}_{2}$, the larger bond length change of Mo-Te bonds in $1 \mathrm{~T}^{\prime}-\mathrm{MoTe}_{2}$ after the iodine-assistance may also help to stabilize the $1 \mathrm{~T}^{\prime}$ phase. Actually, in consideration of the different structures of TMDs, assisted media which can stabilize the internal lattice strain are essential to phase-selective synthesis for metastable TMDs.

\section{SUMMARY AND OUTLOOK}

Phase engineering of $2 \mathrm{D}$ TMDs is essential to both structural engineering and property modulation, which arouse worldwide concern. Enormous efforts have been made to develop phase engineering strategies with high efficiency and excellent controllability. To obtain specific phases of TMDs, it is necessary to develop controllable phase engineering strategies which are classified into the phase transition and phase-selective synthesis strategies. Phase transition can be achieved through intercalation, charge transfer, external irradiation, thermal treatment and stress induction, varying in efficiency, controllability and phase purity. Currently, phase-selective synthesis strategies, such as precursor design, temperature control, atmosphere regulation and medium assistance have been utilized to directly generate specific phase TMDs and heterostructures. Their excellent selectivity and high phase purity can promote further development of device construction and innovative discovery of physical phenomena.

In spite of the recent progress made in phase engineering, challenges still remain to be overcome. Initially, phase purity is vital to both the transformation behavior of structure and property and the controllable synthesis of TMDs with specific phases. The phase transition induced by lithium ion intercalation for $\mathrm{MoS}_{2}$ exhibits a $\sim 80 \%$ phase purity from $2 \mathrm{H}$ to $1 \mathrm{~T}$ phase [119]. In addition, the impurities or the defects introduced during phase engineering process can also affect the phase purity. Secondly, the stability of the products is also an important issue. The as-obtained metastable phases may convert to the original stable ones. Hence, it is 
necessary to stabilize the products by post-treating or introducing stabilizing media. However, such post treatments and nonvolatile stabilizing media would result in the unexpected doping or defects and even the sacrifice of intrinsic properties. Finally, phase engineering for other TMDs (e.g., group VB TMDs) or new phases still remain to be uncovered.

Phase engineering is acknowledged as a promising field for structural and property modulation, as well as the enrichment of the phase library of 2D TMDs. Actually, there may be distinct phases from the existing ones, which exhibit extremely special properties. We are convinced that phase engineering of 2D TMDs especially for the advanced phase-selective synthesis will further promote the expansion of $2 \mathrm{D}$ material family and the development of application technologies.

\section{Received 8 December 2018; accepted 15 January 2019;} published online 13 February 2019

1 Zeng M, Xiao Y, Liu J, et al. Exploring two-dimensional materials toward the next-generation circuits: from monomer design to assembly control. Chem Rev, 2018, 118: 6236-6296

2 Yang H, Kim SW, Chhowalla M, et al. Structural and quantumstate phase transition in van der Waals layered materials. Nat Phys, 2017, 13: 931-937

3 Cai Z, Liu B, Zou X, et al. Chemical vapor deposition growth and applications of two-dimensional materials and their heterostructures. Chem Rev, 2018, 118: 6091-6133

4 Li H, Li Y, Aljarb A, et al. Epitaxial growth of two-dimensional layered transition-metal dichalcogenides: growth mechanism, controllability, and scalability. Chem Rev, 2018, 118: 6134-6150

5 Han GH, Duong DL, Keum DH, et al. Van der Waals metallic transition metal dichalcogenides. Chem Rev, 2018, 118: 62976336

6 Tan C, Lai Z, Zhang H. Ultrathin two-dimensional multinary layered metal chalcogenide nanomaterials. Adv Mater, 2017, 29: 1701392

7 Zhang X, Cheng H, Zhang H. Recent progress in the preparation, assembly, transformation, and applications of layer-structured nanodisks beyond graphene. Adv Mater, 2017, 29: 1701704

8 Tan C, Cao X, Wu XJ, et al. Recent advances in ultrathin twodimensional nanomaterials. Chem Rev, 2017, 117: 6225-6331

9 Sun L, Zheng J. Optical visualization of $\mathrm{MoS}_{2}$ grain boundaries by gold deposition. Sci China Mater, 2018, 61: 1154-1158

10 Liu J, Cao H, Jiang B, et al. Newborn 2D materials for flexible energy conversion and storage. Sci China Mater, 2016, 59: 459474

11 Iannaccone G, Bonaccorso F, Colombo L, et al. Quantum engineering of transistors based on 2D materials heterostructures. Nat Nanotechnol, 2018, 13: 183-191

12 Ali MN, Xiong J, Flynn S, et al. Large, non-saturating magnetoresistance in $\mathrm{WTe}_{2}$. Nature, 2014, 514: 205-208

13 Costanzo D, Jo S, Berger H, et al. Gate-induced superconductivity in atomically thin $\mathrm{MoS}_{2}$ crystals. Nat Nanotechnol, 2016, 11:339344

14 Xi X, Wang Z, Zhao W, et al. Ising pairing in superconducting
$\mathrm{NbSe}_{2}$ atomic layers. Nat Phys, 2015, 12: 139-143

15 Costanzo D, Zhang H, Reddy BA, et al. Tunnelling spectroscopy of gate-induced superconductivity in $\mathrm{MoS}_{2}$. Nat Nanotechnol, 2018, 13: 483-488

16 Sohn E, Xi X, He WY, et al. An unusual continuous paramagnetic-limited superconducting phase transition in $2 \mathrm{D} \mathrm{NbSe}{ }_{2}$. Nat Mater, 2018, 17: 504-508

17 Fei Z, Zhao W, Palomaki TA, et al. Ferroelectric switching of a two-dimensional metal. Nature, 2018, 560: 336-339

18 Bonilla M, Kolekar S, Ma Y, et al. Strong room-temperature ferromagnetism in $\mathrm{VSe}_{2}$ monolayers on van der Waals substrates. Nat Nanotechnol, 2018, 13: 289-293

19 Wu S, Fatemi V, Gibson QD, et al. Observation of the quantum spin Hall effect up to 100 kelvin in a monolayer crystal. Science, 2018, 359: 76-79

20 Tang S, Zhang C, Wong D, et al. Quantum spin Hall state in monolayer 1T'-WTe 2 . Nat Phys, 2017, 13: 683-687

21 Voiry D, Mohite A, Chhowalla M. Phase engineering of transition metal dichalcogenides. Chem Soc Rev, 2015, 44: 2702-2712

22 Ma Y, Kuc A, Jing Y, et al. Two-dimensional Haeckelite $\mathrm{NbS}_{2}$ : A diamagnetic high-mobility semiconductor with $\mathrm{Nb}^{4+}$ ions. Angew Chem Int Ed, 2017, 56: 10214-10218

23 Ma X, Guo P, Yi C, et al. Raman scattering in the transition-metal dichalcogenides of $1 \mathrm{~T}^{\prime}-\mathrm{MoTe}_{2}, \mathrm{~T}_{\mathrm{d}}-\mathrm{MoTe}_{2}$, and $\mathrm{T}_{\mathrm{d}}-\mathrm{WTe}_{2}$. Phys Rev B, 2016, 94: 214105

24 Shirodkar SN, Waghmare UV. Emergence of ferroelectricity at a metal-semiconductor transition in a 1T monolayer of $\mathrm{MoS}_{2}$. Phys Rev Lett, 2014, 112: 157601

25 Chhowalla M, Shin HS, Eda G, et al. The chemistry of twodimensional layered transition metal dichalcogenide nanosheets. Nat Chem, 2013, 5: 263-275

26 Wilson JA, Yoffe AD. The transition metal dichalcogenides discussion and interpretation of the observed optical, electrical and structural properties. Adv Phys, 1969, 18: 193-335

$27 \mathrm{Xu} \mathrm{M}$, Liang $\mathrm{T}$, Shi $\mathrm{M}$, et al. Graphene-like two-dimensional materials. Chem Rev, 2013, 113: 3766-3798

28 Ataca C, Şahin H, Ciraci S. Stable, single-layer $\mathrm{MX}_{2}$ transitionmetal oxides and dichalcogenides in a honeycomb-like structure. J Phys Chem C, 2012, 116: 8983-8999

29 Zhang X, Lai Z, Ma Q, et al. Novel structured transition metal dichalcogenide nanosheets. Chem Soc Rev, 2018, 47: 3301-3338

30 Wang X, Song Z, Wen W, et al. Potential 2D materials with phase transitions: structure, synthesis, and device applications. Adv Mater, 2018, 499: 1804682

31 Wang J, Wei Y, Li H, et al. Crystal phase control in two-dimensional materials. Sci China Chem, 2018, 61: 1227-1242

32 Zhou J, Lin J, Huang X, et al. A library of atomically thin metal chalcogenides. Nature, 2018, 556: 355-359

33 Duerloo KAN, Li Y, Reed EJ. Structural phase transitions in twodimensional Mo- and W-dichalcogenide monolayers. Nat Commun, 2014, 5: 4214

34 Cheng P, Sun K, Hu YH. Memristive behavior and ideal memristor of $1 \mathrm{~T}$ phase $\mathrm{MoS}_{2}$ nanosheets. Nano Lett, 2016, 16: 572-576

35 Lin C, Zhu X, Feng J, et al. Hydrogen-incorporated $\mathrm{TiS}_{2}$ ultrathin nanosheets with ultrahigh conductivity for stamp-transferrable electrodes. J Am Chem Soc, 2013, 135: 5144-5151

36 Li LJ, O'Farrell ECT, Loh KP, et al. Controlling many-body states by the electric-field effect in a two-dimensional material. Nature, 2016, 529: 185-189

37 Chen P, Pai WW, Chan YH, et al. Emergence of charge density 
waves and a pseudogap in single-layer $\mathrm{TiTe}_{2}$. Nat Commun, 2017, 8: 516

38 Li L, Fang X, Zhai T, et al. Electrical transport and high-performance photoconductivity in individual $\mathrm{ZrS}_{2}$ nanobelts. $\mathrm{Adv}$ Mater, 2010, 22: 4151-4156

39 Mleczko MJ, Zhang C, Lee $\mathrm{HR}$, et al. $\mathrm{HfSe}_{2}$ and $\mathrm{ZrSe}_{2}$ : Twodimensional semiconductors with native high- $\kappa$ oxides. Sci Adv, 2017, 3: e1700481

40 Tsipas P, Tsoutsou D, Fragkos S, et al. Massless dirac fermions in $\mathrm{ZrTe}_{2}$ semimetal grown on InAs(111) by van der Waals epitaxy. ACS Nano, 2018, 12: 1696-1703

41 Fu L, Wang F, Wu B, et al. Van der Waals epitaxial growth of atomic layered $\mathrm{HfS}_{2}$ crystals for ultrasensitive near-infrared phototransistors. Adv Mater, 2017, 29: 1700439

42 Mangelsen S, Naumov PG, Barkalov OI, et al. Large nonsaturating magnetoresistance and pressure-induced phase transition in the layered semimetal $\mathrm{HfTe}_{2}$. Phys Rev B, 2017, 96: 205148

43 Guo Y, Deng H, Sun X, et al. Modulation of metal and insulator states in $2 \mathrm{D}$ ferromagnetic $\mathrm{VS}_{2}$ by van der Waals interaction engineering. Adv Mater, 2017, 29: 1700715

44 Ji Q, Li C, Wang J, et al. Metallic vanadium disulfide nanosheets as a platform material for multifunctional electrode applications. Nano Lett, 2017, 17: 4908-4916

45 Li J, Zhao B, Chen P, et al. Synthesis of ultrathin metallic $\mathrm{MTe}_{2}$ $(\mathrm{M}=\mathrm{V}, \mathrm{Nb}, \mathrm{Ta})$ single-crystalline nanoplates. Adv Mater, 2018, 30: 1801043

46 Wang $\mathrm{X}$, Lin J, Zhu Y, et al. Chemical vapor deposition of trigonal prismatic $\mathrm{NbS}_{2}$ monolayers and 3R-polytype few-layers. Nanoscale, 2017, 9: 16607-16611

47 Han JH, Kim HK, Baek B, et al. Activation of the basal plane in two dimensional transition metal chalcogenide nanostructures. J Am Chem Soc, 2018, 140: 13663-13671

48 Calandra M. Phonon-assisted magnetic mott-insulating state in the charge density wave phase of single-layer $1 \mathrm{~T} \mathrm{NbSe} \mathrm{N}_{2}$. Phys Rev Lett, 2018, 121: 026401

49 Yoshida M, Ye J, Zhang Y, et al. Extended polymorphism of twodimensional material. Nano Lett, 2017, 17: 5567-5571

50 Wang $\mathrm{X}$, Liu $\mathrm{H}$, Wu J, et al. Chemical growth of $1 \mathrm{~T}-\mathrm{TaS}_{2}$ monolayer and thin films: robust charge density wave transitions and high bolometric responsivity. Adv Mater, 2018, 30: 1800074

51 Shi J, Chen X, Zhao L, et al. Chemical vapor deposition grown wafer-scale 2D tantalum diselenide with robust charge-densitywave order. Adv Mater, 2018, 30: 1804616

52 Miller DC, Mahanti SD, Duxbury PM. Charge density wave states in tantalum dichalcogenides. Phys Rev B, 2018, 97: 045133

53 Fu L, Sun Y, Wu N, et al. Direct growth of $\mathrm{MoS}_{2} / \mathrm{h}-\mathrm{BN}$ heterostructures via a sulfide-resistant alloy. ACS Nano, 2016, 10: 20632070

54 Acerce M, Voiry D, Chhowalla M. Metallic 1T phase $\mathrm{MoS}_{2}$ nanosheets as supercapacitor electrode materials. Nat Nanotechnol, 2015, 10: 313-318

55 Tongay S, Zhou J, Ataca C, et al. Thermally driven crossover from indirect toward direct bandgap in 2D semiconductors: $\mathrm{MoSe}_{2}$ versus $\mathrm{MoS}_{2}$. Nano Lett, 2012, 12: 5576-5580

56 Yu Y, Nam GH, He Q, et al. High phase-purity $1 \mathrm{~T}^{\prime}-\mathrm{MoS}_{2}$ - and $1 \mathrm{~T}$ '-MoSe $\mathrm{M}_{2}$-layered crystals. Nat Chem, 2018, 10: 638-643

57 Sung JH, Heo H, Si S, et al. Coplanar semiconductor-metal circuitry defined on few-layer $\mathrm{MoTe}_{2}$ via polymorphic heteroepitaxy. Nat Nanotechnol, 2017, 12: 1064-1070
58 Zhang Q, Xiao Y, Zhang T, et al. Iodine-mediated chemical vapor deposition growth of metastable transition metal dichalcogenides. Chem Mater, 2017, 29: 4641-4644

59 Deng K, Wan G, Deng P, et al. Experimental observation of topological Fermi arcs in type-II Weyl semimetal $\mathrm{MoTe}_{2}$. Nat Phys, 2016, 12: 1105-1110

60 Gao Y, Liu Z, Sun DM, et al. Large-area synthesis of high-quality and uniform monolayer $\mathrm{WS}_{2}$ on reusable Au foils. Nat Commun, 2015, 6: 8569

61 Wang QH, Kalantar-Zadeh K, Kis A, et al. Electronics and optoelectronics of two-dimensional transition metal dichalcogenides. Nat Nanotechnol, 2012, 7: 699-712

62 Ma Y, Liu B, Zhang A, et al. Reversible semiconducting-to-metallic phase transition in chemical vapor deposition grown monolayer $\mathrm{WSe}_{2}$ and applications for devices. ACS Nano, 2015, 9: 7383-7391

63 Zheng F, Cai C, Ge S, et al. On the quantum spin hall gap of monolayer 1T'-WTe 2 . Adv Mater, 2016, 28: 4845-4851

64 Wang Y, Liu E, Liu H, et al. Gate-tunable negative longitudinal magnetoresistance in the predicted type-II Weyl semimetal WTe $\mathrm{W}_{2}$. Nat Commun, 2016, 7: 13142

65 Tongay S, Sahin H, Ko C, et al. Monolayer behaviour in bulk $\mathrm{ReS}_{2}$ due to electronic and vibrational decoupling. Nat Commun, 2014, 5: 3252

66 Zhong HX, Gao S, Shi JJ, et al. Quasiparticle band gaps, excitonic effects, and anisotropic optical properties of the monolayer distorted $1 \mathrm{~T}$ diamond-chain structures $\mathrm{ReS}_{2}$ and $\mathrm{ReSe}_{2}$. Phys Rev B, 2015, 92: 115438

67 Miró P, Ghorbani-Asl M, Heine T. Two dimensional materials beyond $\mathrm{MoS}_{2}$ : Noble-transition-metal dichalcogenides. Angew Chem Int Ed, 2014, 53: 3015-3018

68 Zhao Y, Qiao J, Yu Z, et al. High-electron-mobility and air-stable 2D layered $\mathrm{PtSe}_{2}$ FETs. Adv Mater, 2017, 29: 1604230

69 Fu L, Hu D, Mendes RG, et al. Highly organized epitaxy of dirac semimetallic $\mathrm{PtTe}_{2}$ crystals with extrahigh conductivity and visible surface plasmons at edges. ACS Nano, 2018, 12: 9405-9411

70 Py MA, Haering RR. Structural destabilization induced by lithium intercalation in $\mathrm{MoS}_{2}$ and related compounds. Can J Phys, 1983, 61: 76-84

71 Yang D, Sandoval SJ, Divigalpitiya WMR, et al. Structure of single-molecular-layer $\mathrm{MoS}_{2}$. Phys Rev B, 1991, 43: 12053-12056

72 Wang $\mathrm{H}, \mathrm{Lu} \mathrm{Z}, \mathrm{Xu} \mathrm{S}$, et al. Electrochemical tuning of vertically aligned $\mathrm{MoS}_{2}$ nanofilms and its application in improving hydrogen evolution reaction. Proc Natl Acad Sci USA, 2013, 110: 19701-19706

73 Qi $\mathrm{Y}, \mathrm{Xu} \mathrm{Q}$, Wang $\mathrm{Y}$, et al. $\mathrm{CO}_{2}$-induced phase engineering: protocol for enhanced photoelectrocatalytic performance of $2 \mathrm{D}$ $\mathrm{MoS}_{2}$ nanosheets. ACS Nano, 2016, 10: 2903-2909

74 Zhang J, Wu J, Guo $\mathrm{H}$, et al. Unveiling active sites for the hydrogen evolution reaction on monolayer $\mathrm{MoS}_{2}$. Adv Mater, 2017, 29: 1701955

75 Yamaguchi H, Blancon JC, Kappera R, et al. Spatially resolved photoexcited charge-carrier dynamics in phase-engineered monolayer $\mathrm{MoS}_{2}$. ACS Nano, 2015, 9: 840-849

76 Kappera R, Voiry D, Yalcin SE, et al. Phase-engineered low-resistance contacts for ultrathin $\mathrm{MoS}_{2}$ transistors. Nat Mater, 2014, 13: $1128-1134$

77 Silbernagel BG. Lithium intercalation complexes of layered transition metal dichalcogenides: An NMR survey of physical properties. Solid State Commun, 1975, 17: 361-365 
78 Tan C, Luo Z, Chaturvedi A, et al. Preparation of high-percentage 1T-phase transition metal dichalcogenide nanodots for electrochemical hydrogen evolution. Adv Mater, 2018, 30: 1705509

79 Tan C, Zhao W, Chaturvedi A, et al. Preparation of single-layer $\mathrm{MoS}_{2 x} \mathrm{Se}_{2(1-x)}$ and $\mathrm{Mo}_{x} \mathrm{~W}_{1-x} \mathrm{~S}_{2}$ nanosheets with high-concentration metallic $1 \mathrm{~T}$ phase. Small, 2016, 12: 1866-1874

80 Zeng Z, Yin Z, Huang X, et al. Single-layer semiconducting nanosheets: high-yield preparation and device fabrication. Angew Chem Int Ed, 2011, 50: 11093-11097

81 Lai Z, Chaturvedi A, Wang Y, et al. Preparation of 1T'-phase $\operatorname{ReS}_{2 x} \mathrm{Se}_{2(1-x)}(x=0-1)$ nanodots for highly efficient electrocatalytic hydrogen evolution reaction. J Am Chem Soc, 2018, 140: 85638568

82 Fan X, Xu P, Zhou D, et al. Fast and efficient preparation of exfoliated $2 \mathrm{H} \mathrm{MoS}_{2}$ nanosheets by sonication-assisted lithium intercalation and infrared laser-induced $1 \mathrm{~T}$ to $2 \mathrm{H}$ phase reversion. Nano Lett, 2015, 15: 5956-5960

83 Cheng Y, Nie A, Zhang Q, et al. Origin of the phase transition in lithiated molybdenum disulfide. ACS Nano, 2014, 8: 1144711453

84 Yan S, Qiao W, He X, et al. Enhancement of magnetism by structural phase transition in $\mathrm{MoS}_{2}$. Appl Phys Lett, 2015, 106: 012408

85 Zhao W, Ribeiro RM, Eda G. Electronic structure and optical signatures of semiconducting transition metal dichalcogenide nanosheets. Acc Chem Res, 2015, 48: 91-99

86 Yin Y, Han J, Zhang Y, et al. Contributions of phase, sulfur vacancies, and edges to the hydrogen evolution reaction catalytic activity of porous molybdenum disulfide nanosheets. J Am Chem Soc, 2016, 138: 7965-7972

87 Eda G, Yamaguchi H, Voiry D, et al. Photoluminescence from chemically exfoliated $\mathrm{MoS}_{2}$. Nano Lett, 2011, 11: 5111-5116

88 Tan SJR, Abdelwahab I, Ding Z, et al. Chemical stabilization of 1T' phase transition metal dichalcogenides with giant optical Kerr nonlinearity. J Am Chem Soc, 2017, 139: 2504-2511

89 Sun L, Yan X, Zheng J, et al. Layer-dependent chemically induced phase transition of two-dimensional $\mathrm{MoS}_{2}$. Nano Lett, 2018, 18: 3435-3440

90 Wang L, Xu Z, Wang W, et al. Atomic mechanism of dynamic electrochemical lithiation processes of $\mathrm{MoS}_{2}$ nanosheets. J Am Chem Soc, 2014, 136: 6693-6697

91 Kan M, Wang JY, Li XW, et al. Structures and phase transition of a $\mathrm{MoS}_{2}$ monolayer. J Phys Chem C, 2014, 118: 1515-1522

92 Wang X, Shen X, Wang Z, et al. Atomic-scale clarification of structural transition of $\mathrm{MoS}_{2}$ upon sodium intercalation. ACS Nano, 2014, 8: 11394-11400

93 Zhang R, Tsai IL, Chapman J, et al. Superconductivity in potassium-doped metallic polymorphs of $\mathrm{MoS}_{2}$. Nano Lett, 2016, 16: 629-636

94 Liu Q, Li X, He Q, et al. Gram-scale aqueous synthesis of stable few-layered $1 \mathrm{~T}-\mathrm{MoS}_{2}$ : Applications for visible-light-driven photocatalytic hydrogen evolution. Small, 2015, 11: 5556-5564

95 Liu Q, Li X, Xiao Z, et al. Stable metallic 1T-WS 2 nanoribbons intercalated with ammonia ions: the correlation between structure and electrical/optical properties. Adv Mater, 2015, 27: 48374844

96 Li Y, Duerloo KAN, Wauson K, et al. Structural semiconductorto-semimetal phase transition in two-dimensional materials induced by electrostatic gating. Nat Commun, 2016, 7: 10671

97 Wang Y, Xiao J, Zhu H, et al. Structural phase transition in monolayer $\mathrm{MoTe}_{2}$ driven by electrostatic doping. Nature, 2017, 550: 487-491

98 Kim S, Song S, Park J, et al. Long-range lattice engineering of $\mathrm{MoTe}_{2}$ by a 2D electride. Nano Lett, 2017, 17: 3363-3368

99 Kang Y, Najmaei S, Liu Z, et al. Plasmonic hot electron induced structural phase transition in a $\mathrm{MoS}_{2}$ monolayer. Adv Mater, 2014, 26: 6467-6471

100 Enyashin AN, Yadgarov L, Houben L, et al. New route for stabilization of 1T-WS 2 and $\mathrm{MoS}_{2}$ phases. J Phys Chem C, 2011, 115: 24586-24591

101 Chen Y, Xi J, Dumcenco DO, et al. Tunable band gap photoluminescence from atomically thin transition-metal dichalcogenide alloys. ACS Nano, 2013, 7: 4610-4616

102 Lin YC, Dumcenco DO, Huang YS, et al. Atomic mechanism of the semiconducting-to-metallic phase transition in single-layered $\mathrm{MoS}_{2}$. Nat Nanotech, 2014, 9: 391-396

103 Suh J, Tan TL, Zhao W, et al. Reconfiguring crystal and electronic structures of $\mathrm{MoS}_{2}$ by substitutional doping. Nat Commun, 2018, 9: 199

104 Title RS, Shafer MW. Band structure of the layered transitionmetal dichalcogenides: an experimental study by electron paramagnetic resonance on Nb-doped $\mathrm{MoS}_{2}$. Phys Rev Lett, 1972, 28: 808-810

105 Zhu J, Wang Z, Yu H, et al. Argon plasma induced phase transition in monolayer $\mathrm{MoS}_{2}$. J Am Chem Soc, 2017, 139: 1021610219

106 Cho S, Kim S, Kim JH, et al. Phase patterning for ohmic homojunction contact in $\mathrm{MoTe}_{2}$. Science, 2015, 349: 625-628

107 Chow PK, Jacobs-Gedrim RB, Gao J, et al. Defect-induced photoluminescence in monolayer semiconducting transition metal dichalcogenides. ACS Nano, 2015, 9: 1520-1527

108 Tosun M, Chan L, Amani M, et al. Air-stable n-doping of $\mathrm{WSe}_{2}$ by anion vacancy formation with mild plasma treatment. ACS Nano, 2016, 10: 6853-6860

109 Park JC, Yun SJ, Kim H, et al. Phase-engineered synthesis of centimeter-scale $1 \mathrm{~T}^{\prime}$ - and $2 \mathrm{H}$-molybdenum ditelluride thin films. ACS Nano, 2015, 9: 6548-6554

110 Keum DH, Cho S, Kim JH, et al. Bandgap opening in few-layered monoclinic $\mathrm{MoTe}_{2}$. Nat Phys, 2015, 11: 482-486

111 Wang Z, Sun YY, Abdelwahab I, et al. Surface-limited superconducting phase transition on $1 \mathrm{~T}-\mathrm{TaS}_{2}$. ACS Nano, 2018, 12: 12619-12628

112 Song S, Keum DH, Cho S, et al. Room temperature semiconductor-metal transition of $\mathrm{MoTe}_{2}$ thin films engineered by strain. Nano Lett, 2016, 16: 188-193

113 Nayak AP, Pandey T, Voiry D, et al. Pressure-dependent optical and vibrational properties of monolayer molybdenum disulfide. Nano Lett, 2015, 15: 346-353

114 Voiry D, Goswami A, Kappera R, et al. Covalent functionalization of monolayered transition metal dichalcogenides by phase engineering. Nat Chem, 2014, 7: 45-49

115 Yang K, Wang X, Li H, et al. Composition- and phase-controlled synthesis and applications of alloyed phase heterostructures of transition metal disulphides. Nanoscale, 2017, 9: 5102-5109

116 Kang K, Xie S, Huang L, et al. High-mobility three-atom-thick semiconducting films with wafer-scale homogeneity. Nature, 2015, 520: 656-660

117 Najmaei S, Liu Z, Zhou W, et al. Vapour phase growth and grain boundary structure of molybdenum disulphide atomic layers. Nat Mater, 2013, 12: 754-759 
Ju M, Liang X, Liu J, et al. Universal substrate-trapping strategy to grow strictly monolayer transition metal dichalcogenides crystals. Chem Mater, 2017, 29: 6095-6103

119 Liu L, Wu J, Wu L, et al. Phase-selective synthesis of $1 \mathrm{~T}^{\prime} \mathrm{MoS}_{2}$ monolayers and heterophase bilayers. Nat Mater, 2018, 17: 11081114

120 Zhou L, Xu K, Zubair A, et al. Large-area synthesis of high-quality uniform few-layer $\mathrm{MoTe}_{2}$. J Am Chem Soc, 2015, 137: 1189211895

121 Zhou L, Zubair A, Wang Z, et al. Synthesis of high-quality largearea homogenous $1 \mathrm{~T}^{\prime} \mathrm{MoTe}_{2}$ from chemical vapor deposition. Adv Mater, 2016, 28: 9526-9531

122 Yoo Y, DeGregorio ZP, Su Y, et al. In-plane 2H-1T' $\mathrm{MoTe}_{2}$ homojunctions synthesized by flux-controlled phase engineering. Adv Mater, 2017, 29: 1605461

123 Chen P, Pai WW, Chan YH, et al. Large quantum-spin-Hall gap in single-layer 1T' $\mathrm{WSe}_{2}$. Nat Commun, 2018, 9: 2003

124 Li S, Wang S, Tang DM, et al. Halide-assisted atmospheric pressure growth of large $\mathrm{WSe}_{2}$ and $\mathrm{WS}_{2}$ monolayer crystals. Appl Mater Today, 2015, 1: 60-66

125 Chen K, Chen Z, Wan X, et al. A simple method for synthesis of high-quality millimeter-scale 1T' transition-metal telluride and near-field nanooptical properties. Adv Mater, 2017, 29: 1700704

Acknowledgements This work was supported by the National Natural Science Foundation of China (21673161 and 21473124), the Science and Technology Department of Hubei Province (2017AAA114), and the Sino-German Center for Research Promotion (1400).

Author contributions Xiao $\mathrm{Y}$, Zhou M, Liu J and $\mathrm{Xu} J$ wrote the manuscript; Fu L and Xiao Y developed the concept and revised the manuscript; Xiao Y prepared the figures; Xiao Y and Liu J classified and analyzed the reference papers. All authors participated in the general discussion.

Conflict of interest The authors declare no conflict of interest. 

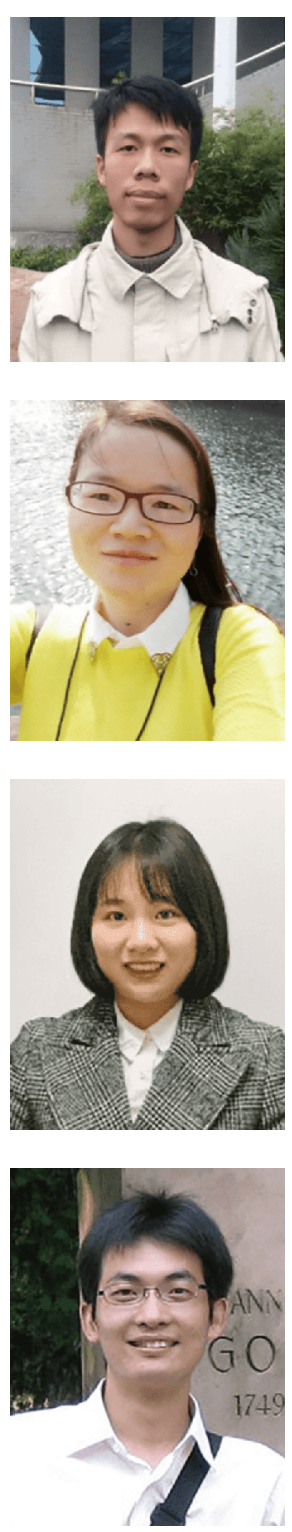

Yao Xiao received his BSc degree in chemistry from Wuhan University in 2016 and continued as a $\mathrm{PhD}$ candidate under the supervision of Prof. Lei Fu at the Institute of Advanced Studies at Wuhan University. His current scientific interest focuses on the special growing phenomena and electronics of 2D materials and heterostructures.

Mengyue Zhou received her BSc from Huazhong Agricultural University in 2017 and continued her studies as a master candidate under the supervision of Prof. Lei Fu at the College of Chemistry and Molecular Sciences at Wuhan University. Her current research interest is the controllable growth of two-dimensional material.

Jinglu Liu is pursuing her Bachelor's degree from the College of Chemistry and Molecular Science, Wuhan University. Her current research focuses on the synthesis of $2 \mathrm{D}$ transition metal dichalcogenide materials.
Lei Fu received his BSc degree in chemistry from Wuhan University in 2001. He obtained his PhD degree from the Institute of Chemistry, Chinese Academy of Sciences in 2006. Then he worked as a Director's Postdoctoral Fellow at the Los Alamos National Laboratory, Los Alamos, NM (2006-2007). Thereafter, he became an Associate Professor at Peking University. In 2012, he joined Wuhan University as a Full Professor. His current interest of research includes the controlled growth and novel property exploration of $2 \mathrm{D}$ atomic layer thin crystals.

\section{二维过渡金属二硫族化合物的相工程}

肖遥, 周梦月 ${ }^{2}$, 刘晶璐 ${ }^{2}$, 徐婧 $^{2}$, 付磊 ${ }^{1,2^{*}}$

摘要 二维过渡金属二硫族化合物因其多样的原子排布和能带结构而备受关注. 除了热力学稳定的相之外, 许多亚稳态相的过渡金属二 硫族化合物也表现出有趣的性质. 为了获得特定相的二维过渡金属二硫族化合物, 相工程策略(包括相转变和相选择合成) 凸显的十分重 要. 在本文中, 我们首先介绍了不同相的二维过渡金属二硫族化合物的结构和稳定性. 接着, 我们总结了多种相转变策略的详细过程和机 理. 此外, 由于对过渡金属二硫族化合物相纯度的需求不断提升, 我们也展示了新型的相选择合成策略. 最后, 我们从相纯度和可控性两个 方面指出二维过渡金属二硫族化合物相工程面临的挑战, 展望了相工程策略在可控获得高相纯度过渡金属二硫族化合物的前景. 这篇综 述将促进二维过渡金属二硫族化合物及其他二维材料的可控相工程在基础研究和实际应用方面的发展. 